\title{
A HYPERGRAPH PARTITIONING MODEL FOR PROFILE MINIMIZATION*
}

\author{
SEHER ACER ${ }^{\dagger}$, ENVER KAYAASLAN ${ }^{\dagger \ddagger}$, AND CEVDET AYKANAT ${ }^{\dagger}$
}

\begin{abstract}
In this paper, the aim is to symmetrically permute the rows and columns of a given sparse symmetric matrix so that the profile of the permuted matrix is minimized. We formulate this permutation problem by first defining the $m$-way ordered hypergraph partitioning (moHP) problem and then showing the correspondence between profile minimization and moHP problems. For solving the moHP problem, we propose a recursive-bipartitioning-based hypergraph partitioning algorithm, which we refer to as the moHP algorithm. This algorithm achieves a linear part ordering via left-toright bipartitioning. In this algorithm, we utilize fixed vertices and two novel cut-net manipulation techniques in order to address the minimization objective of the moHP problem. We show the correctness of the moHP algorithm and describe how the existing partitioning tools can be utilized for its implementation. Experimental results on an extensive set of matrices show that the moHP algorithm obtains a smaller profile than the state-of-the-art profile reduction algorithms, which then results in considerable improvements in the factorization runtime in a direct solver.
\end{abstract}

Key words. sparse matrices, matrix ordering, matrix profile, matrix envelope, profile minimization, profile reduction, hypergraph partitioning, recursive bipartitioning

AMS subject classifications. 05C50, 05C85, 65F05, 65F50, 68R 10

DOI. $10.1137 / 17 \mathrm{M} 1161245$

1. Introduction. The focus of this work is to minimize the envelope size, i.e., profile, of a given $m \times m$ sparse symmetric matrix $A=\left(a_{i j}\right)$ through symmetric row/column permutation. The envelope of $A, E(A)$, is defined as the set of index pairs in each row that lie between the first nonzero entry and the diagonal. That is,

$$
E(A)=\{(i, j): f c(i) \leq j<i, i=1,2, \ldots, m\}
$$

where $f c(i)$ denotes the column index of the first nonzero entry in row $i$, i.e., $f c(i)=$ $\min \left\{j: a_{i j} \neq 0\right\}$. The size of the envelope of $A$ is referred to as the profile of $A$, which is denoted by $P(A)$. Note that the profile can also be expressed as the sum of row widths in the envelope, that is,

$$
P(A)=|E(A)|=\sum_{i=1}^{m}(i-f c(i)) .
$$

Díaz, Petit, and Serna [16] describe a number of graph layout problems which are similar or equivalent to the profile minimization problem and the application areas of these problems.

The profile minimization problem arises in various applications. The greatest attention given to this problem is from the scientific computing domain due to improving the performance of the sparse solvers. Basically, sparse Gaussian elimination benefits from an ordering of the input matrix with small profile in terms of both

*Submitted to the journal's Methods and Algorithms for Scientific Computing section December 14, 2017; accepted for publication (in revised form) October 12, 2018; published electronically January $2,2019$.

http://www.siam.org/journals/sisc/41-1/M116124.html

†Computer Engineering Department, Bilkent University, 06800, Ankara, Turkey (acer@cs.bilkent. edu.tr, enver@cs.bilkent.edu.tr, aykanat@cs.bilkent.edu.tr).

${ }^{\ddagger}$ Currently with Google Switzerland, 8002, Zürich, Switzerland. 
storage and number of floating-point operations [20, 37]. The computational complexity of envelope methods is proportional to the sum of squares of row widths. Similarly, the computational complexity of frontal methods is proportional to the sum of squares of front sizes, where the sum of the profile and the number of rows gives the sum of the front sizes. While envelope methods are now outdated, frontal methods and their extensions, such as multifrontal methods, are still actively used. Davis, Rajamanickam,and Sid-Lakhdar [14] list these methods in their recent and extensive survey on sparse direct methods. Besides direct methods, small profile is also shown to be desirable for improving the performance of iterative methods, including incomplete factorization preconditioners [11, 15, 19, 24, 39]. Furthermore, improving cache hit rates in sparse matrix computations can be considered as another application for this problem $[8,41]$. In addition to the scientific computing domain, the profile metric and the corresponding minimization problem are found to be useful in applications from other domains such as bioinformatics, model checking, and visualization $[5,6,26,28,33,34]$.

The profile minimization problem is NP-hard [32]. Heuristics proposed for solving this problem are plentiful in the literature. In the following, we summarize the most commonly used profile reduction heuristics and refer the reader to the recent systematic review in [4] for a more complete list. The earliest methods such as RCM [21], GPS [23], Gibbs-King [22], and Sloan [40] exploit the level structure obtained on the standard graph representation of the given matrix. Most of the successor methods use a spectral approach [3], which obtains better results compared to the earlier methods at the expense of higher ordering runtimes. These runtimes are improved by hybrid methods [7, 29, 30, 35], which exploit both graph-based and spectral approaches in a multilevel framework. These algorithms include the one proposed by $\mathrm{Hu}$ and Scott [29], which obtains smaller profile values than the preceding algorithms. Reid and Scott [36] show that applying Hager's exchange methods [27] as a postprocessing step to the algorithm proposed by $\mathrm{Hu}$ and Scott [29] achieves even better results.

The contributions of this paper are as follows. We first define an ordered version of the hypergraph partitioning (HP) problem, which we refer to as the $m$-way ordered hypergraph partitioning (moHP) problem. Then, we formulate the profile minimization problem as an moHP problem. To the best of our knowledge, this work is the first in the literature which formulates the profile minimization using an HP problem. For solving the moHP problem, we propose the moHP algorithm, which is based on the recursive bipartitioning (RB) paradigm. The moHP algorithm achieves a linear part ordering via left-to-right bipartitioning. In order to address the minimization objective of the moHP problem, the moHP algorithm utilizes fixed vertices within the RB framework and two novel cut-net manipulation techniques. We theoretically show that minimizing a cost metric in each RB step corresponds to minimizing the objective of the moHP problem. We also show how existing HP tools can be utilized in the proposed RB-based algorithm.

The rest of the paper is organized as follows. Section 2 provides background information. Section 3 presents the moHP problem and shows its correspondence to the profile minimization problem. Section 4 presents the proposed RB-based algorithm for solving the moHP problem, discusses its correctness, and describes the implementation of the proposed algorithm using existing partitioning tools. Section 5 provides the experimental results in comparison with the state-of-the-art profile reduction algorithms, and section 6 concludes. 
2. Preliminaries. A hypergraph $\mathcal{H}=(\mathcal{V}, \mathcal{N})$ is defined as a set of $n$ vertices $\mathcal{V}=\left\{v_{1}, v_{2}, \ldots, v_{n}\right\}$ and a set of $m$ nets $\mathcal{N}=\left\{n_{1}, n_{2}, \ldots, n_{m}\right\}$. In $\mathcal{H}$, each net $n_{i} \in \mathcal{N}$ connects a subset of vertices in $\mathcal{V}$, which is denoted by $\operatorname{Pins}\left(n_{i}\right)$. The vertices in $\operatorname{Pins}\left(n_{i}\right)$ are also referred to as the pins of $n_{i}$. Each vertex $v_{i} \in \mathcal{V}$ is assigned a weight, which is denoted by $w\left(v_{i}\right)$. Each net $n_{i} \in \mathcal{N}$ is assigned a cost, which is denoted by $c\left(n_{i}\right)$.

$\Pi=\left\{\mathcal{V}_{1}, \mathcal{V}_{2}, \ldots, \mathcal{V}_{K}\right\}$ is a $K$-way partition of $\mathcal{H}$ if the parts in $\Pi$ are nonempty, mutually disjoint, and exhaustive. For a given partition $\Pi$, a net $n_{i}$ is said to connect a part $\mathcal{V}_{k}$ if it has pins in $\mathcal{V}_{k}$, i.e., Pins $\left(n_{i}\right) \cap \mathcal{V}_{k} \neq \emptyset$. Net $n_{i}$ is said to be cut if it connects multiple parts in $\Pi$, and uncut/internal, otherwise. The cutsize of $\Pi$ is defined as the sum of the costs of the cut nets, that is,

$$
\text { cutsize }(\Pi)=\sum_{n_{i} \in \mathcal{N}_{c}} c\left(n_{i}\right),
$$

where $\mathcal{N}_{c}$ denotes the set of cut nets in $\Pi$. The weight $W\left(\mathcal{V}_{k}\right)$ of a part $\mathcal{V}_{k}$ is defined as the sum of the weights of the vertices in $\mathcal{V}_{k}$, i.e., $W\left(\mathcal{V}_{k}\right)=\sum_{v_{i} \in \mathcal{V}_{k}} w\left(v_{i}\right)$.

Given $K$ and $\epsilon$ values, the hypergraph partitioning (HP) problem is defined as the problem of finding a $K$-way partition of a given hypergraph so that the cutsize (1) is minimized and a balance on the weights of the parts is maintained by the constraint

$$
W\left(\mathcal{V}_{k}\right) \leq(1+\epsilon) \frac{\sum_{j=1}^{K} W\left(\mathcal{V}_{j}\right)}{K} \text { for } k=1,2, \ldots, K .
$$

Here, $\epsilon$ denotes the maximum allowable imbalance ratio on the weights of the parts.

The HP problem with fixed vertices is a constrained version of the HP problem where, for each part, a subset of vertices can be preassigned to that part before partitioning in such a way that, at the end of the partitioning, they remain in the parts to which they are preassigned. These vertices are called fixed vertices. The set of vertices which are fixed to part $\mathcal{V}_{k}$ is denoted by $\mathcal{F}_{k}$ for $k=1,2, \ldots, K$. The rest of the vertices are called free vertices as they can be assigned to any part.

If $K=2$, then $\Pi=\left\{\mathcal{V}_{1}, \mathcal{V}_{2}\right\}$ is also referred to as a bipartition. We use $\Pi=$ $\left\langle\mathcal{V}_{L}, \mathcal{V}_{R}\right\rangle$ to denote a bipartition in which the order of the parts is relevant. Here, $\mathcal{V}_{L}$ and $\mathcal{V}_{R}$, respectively, denote the left and right parts. In case of bipartitioning with fixed vertices, $\mathcal{F}_{L}$ and $\mathcal{F}_{R}$ denote the sets of vertices that are fixed to $\mathcal{V}_{L}$ and $\mathcal{V}_{R}$, respectively.

For a given sparse matrix $A$, the row-net hypergraph $\mathcal{H}=(\mathcal{V}, \mathcal{N})[9]$ is formed as follows. As implied by the name, each row $i$ in $A$ is represented by a net $n_{i}$ in $\mathcal{N}$. In a dual manner, each column $j$ in $A$ is represented by a vertex $v_{j}$ in $\mathcal{V}$. For each nonzero entry $a_{i j}$ in $A$, net $n_{i}$ connects vertex $v_{j}$ in $\mathcal{H}$.

3. The $m$-way ordered hypergraph partitioning formulation. In this section, we first define a variant of the HP problem, the moHP problem, and then show how the profile minimization problem can be formulated as an moHP problem.

3.1. The $m$-way ordered hypergraph partitioning (moHP) problem. In the moHP problem, we use a special form of partition which is referred to as $m$ way ordered partition $\left(\Pi_{m o}\right)$. Consider a hypergraph $\mathcal{H}=(\mathcal{V}, \mathcal{N})$ with $m$ vertices, that is, $\mathcal{V}=\left\{v_{1}, v_{2}, \ldots, v_{m}\right\}$. A partition of $\mathcal{H}$ is an $m$-way ordered partition if each part contains exactly one vertex and the parts are subject to an order. We use $\Pi_{m o}=\left\langle\mathcal{V}_{1}, \mathcal{V}_{2}, \ldots, \mathcal{V}_{m}\right\rangle$ to denote an $m$-way ordered partition. Figure 1 displays a sample $m$-way ordered partition of a hypergraph with $m=8$ vertices. In this 


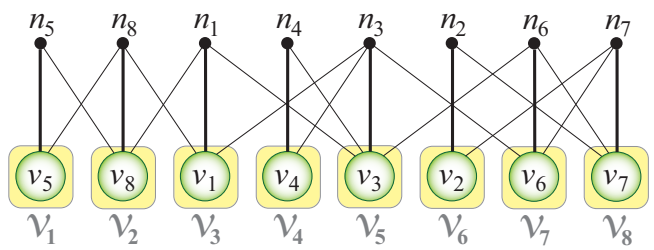

FIG. 1. An $m$-way ordered partition of a hypergraph $\mathcal{H}$ with $m=8$ vertices.

figure, $\mathcal{V}_{1}=\left\{v_{5}\right\}, \mathcal{V}_{2}=\left\{v_{8}\right\}$, and so on. Given an $m$-way ordered partition $\Pi_{m o}$, the position of a vertex $v_{i}, \phi\left(v_{i}\right)$, is defined as the order of the part that contains $v_{i}$. That is, $\phi\left(v_{i}\right)=k$ if and only if $\mathcal{V}_{k}=\left\{v_{i}\right\}$. For example, $\phi\left(v_{1}\right)=3$ in Figure 1 . The leftmost vertex $f_{i}$ of a net $n_{i}$ is defined as the pin of $n_{i}$ with the minimum position. That is,

$$
f_{i}=\arg \min _{v_{j} \in \operatorname{Pins}\left(n_{i}\right)} \phi\left(v_{j}\right) .
$$

For example, $f_{3}=v_{1}$ in Figure 1. The left span of a net $n_{i}, l s\left(n_{i}\right)$, is defined as the difference between the positions of vertices $v_{i}$ and $f_{i}$. That is,

$$
l s\left(n_{i}\right)=\phi\left(v_{i}\right)-\phi\left(f_{i}\right) .
$$

Here, we assume that $v_{i} \in \operatorname{Pins}\left(n_{i}\right)$ for each $n_{i} \in \mathcal{N}$; thus, $l s\left(n_{i}\right)$ is nonnegative. For example, $l s\left(n_{3}\right)=5-3=2$ in Figure 1.

The cost of an $m$-way ordered partition $\Pi_{m o}$ is defined as the sum of the left spans of the nets in $\mathcal{N}$. That is,

$$
\operatorname{cost}\left(\Pi_{m o}\right)=\sum_{n_{i} \in \mathcal{N}} l s\left(n_{i}\right) .
$$

For example, the cost of the $m$-way partition in Figure 1 is 8 . Note that the cost formulation in (4) is quite different from the traditional cutsize definition in (1).

Definition 1 (the moHP problem). Consider a hypergraph $\mathcal{H}=(\mathcal{V}, \mathcal{N})$ with vertex set $\mathcal{V}=\left\{v_{1}, v_{2}, \ldots, v_{m}\right\}$ and net set $\mathcal{N}=\left\{n_{1}, n_{2}, \ldots, n_{m}\right\}$. Assume that $v_{i} \in \operatorname{Pins}\left(n_{i}\right)$ for each net $n_{i} \in \mathcal{N}$. Then, the moHP problem is the problem of finding an $m$-way ordered partition $\Pi_{m o}$ of $\mathcal{H}$ so that the cost given in (4) is minimized.

3.2. Formulation. The following theorem shows how the profile minimization problem can be formulated as an moHP problem.

TheOREM 2. Let $\mathcal{H}(A)=(\mathcal{V}, \mathcal{N})$ be the row-net hypergraph of an $m \times m$ structurally symmetric sparse matrix $A$ with $a_{i i} \neq 0$ for $i=1,2, \ldots, m$. An $m$-way ordered partition $\Pi_{\text {om }}$ of $\mathcal{H}(A)$ can be decoded as a row/column permutation $P$ for $A$ so that minimizing the cost of $\Pi_{m o}$ corresponds to minimizing the profile of the permuted matrix $P A P^{T}$.

Proof. Consider an $m$-way ordered partition $\Pi_{m o}$ of $\mathcal{H}(A)$, which is decoded as a row/column permutation for $A$ in such a way that the order of row/column $i$ in the permuted matrix $P A P^{T}$ is the position $\phi\left(v_{i}\right)$ of vertex $v_{i}$ in $\Pi_{m o}$. That is, the permutation matrix $P$ is formulated as

$$
P=\left[\begin{array}{llll}
p_{1} & p_{2} & \cdots & p_{m}
\end{array}\right]
$$




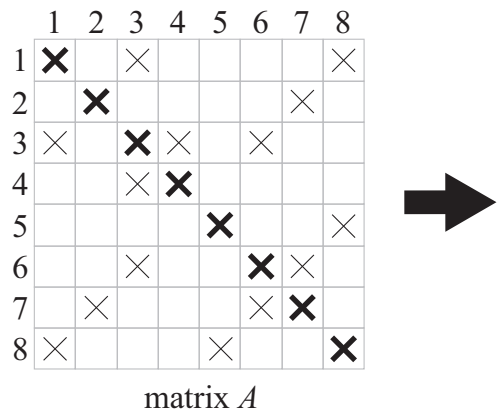

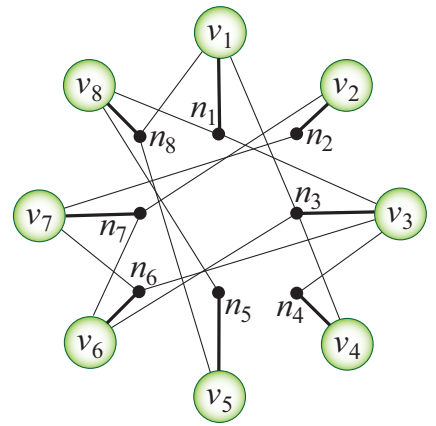

row-net hypergraph $\mathcal{H}(A)$
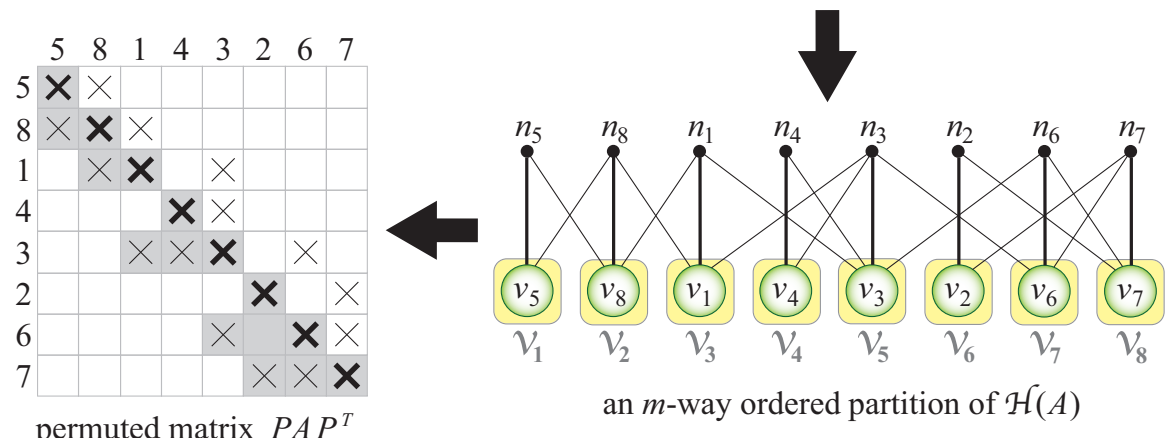

FIG. 2. An illustration for the formulation of the profile minimization problem as an moHP problem.

where $p_{i}$ is a column vector with all zeros except the $\phi\left(v_{i}\right)$ th entry, which is equal to 1 for $i=1,2, \ldots, m$. Consider a row $i$ in $P A P^{T}$. Note that $a_{i i}$ is the $\phi\left(v_{i}\right)$ th diagonal entry of $P A P^{T}$. Let $C_{i}$ denote the set of the columns in which row $i$ has a nonzero entry. By the row-net hypergraph formulation, $v_{j} \in \operatorname{Pins}\left(n_{i}\right)$ if and only if $j \in C_{i}$. Since the order of each column $j \in C_{i}$ in $P A P^{T}$ is set to be $\phi\left(v_{j}\right)$, the column representing vertex $f_{i}$ has the first nonzero entry of row $i$ in $P A P^{T}$. Thus, the contribution of row $i$ to the profile of $P A P^{T}$ is equal to the left span of $n_{i}$ in $\Pi_{m o}$. Hence, the profile of $P A P^{T}$ is equal to the cost of $\Pi_{m o}$. Therefore, minimizing the cost of $\Pi_{m o}$ corresponds to minimizing the profile of $P A P^{T}$.

Figure 2 displays a sample $8 \times 8$ structurally symmetric sparse matrix $A$ with 22 nonzero entries and the row-net hypergraph $\mathcal{H}(A)$ of $A$ with 8 vertices, 8 nets, and 22 pins. The figure also displays an $m$-way ordered partition of $\mathcal{H}(A)$ and the permuted matrix $P A P^{T}$ induced by this partition. For example, consider row 3 in $A$. As seen in the figure, row 3 is ordered as the fifth row in $P A P^{T}$ since $\phi\left(v_{3}\right)=5$. The left span of net $n_{3}$, which represents row 3 , is computed as $l s\left(n_{3}\right)=\phi\left(v_{3}\right)-\phi\left(f_{3}\right)=5-3=2$. Note that the contribution of row 3 to the profile of $P A P^{T}$ is also 2 , which is equal to $l s\left(n_{3}\right)$. The profile of $P A P^{T}$ is 8 , which is equal to the cost of the given $m$-way ordered partition.

4. Recursive-bipartitioning-based moHP algorithm. This section describes the proposed moHP algorithm, which aims at finding an $m$-way ordered partition of a given hypergraph with minimum cost. The moHP algorithm is based on the well-known recursive bipartitioning $(\mathrm{RB})$ paradigm and adopts a left-to-right bipar- 


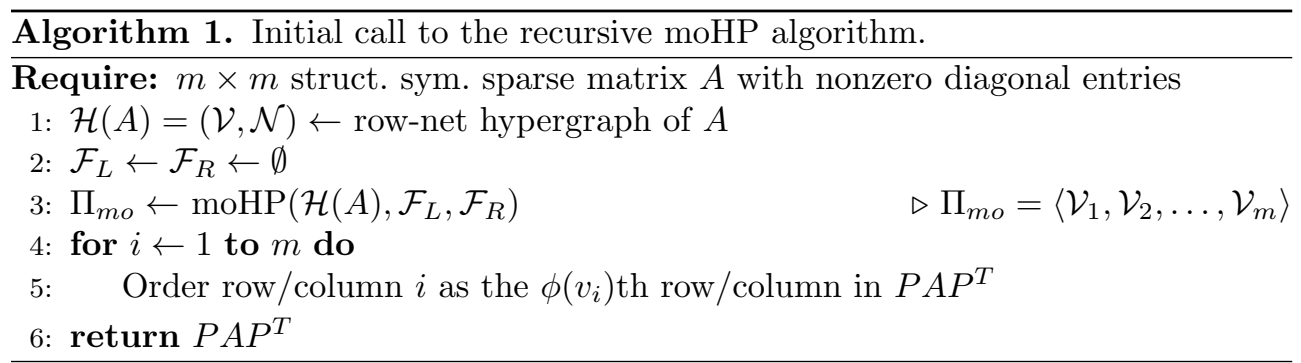

titioning approach. In this approach, a natural order is assumed on the parts of each bipartition, and the final partitions of the left and right parts are combined in such a way that their respective orderings are preserved. Recall that the partitioning cost is defined as the sum of the left spans of the nets in (4). Within the left-to-right bipartitioning approach, the moHP algorithm utilizes fixed vertices in order to target the minimization of these left span values.

4.1. Overall description. Algorithm 1 shows the initial invocation of the recursive moHP algorithm. This algorithm first forms the row-net hypergraph $\mathcal{H}(A)$ of the input $m \times m$ structurally symmetric sparse matrix $A$. In $\mathcal{H}(A)$, each vertex is assigned a unit weight and each net is assigned a unit cost, that is, $w\left(v_{i}\right)=1$ for each $v_{i} \in \mathcal{V}$ and $c\left(n_{i}\right)=1$ for each $n_{i} \in \mathcal{N}$. Then, the moHP algorithm is invoked on $\mathcal{H}(A)$ with empty fixed-vertex sets $F_{L}$ and $\mathcal{F}_{R}$, and at the end of this invocation, an $m$-way ordered partition $\Pi_{m o}$ of $\mathcal{H}(A)$ is returned. $\Pi_{m o}$ is then utilized to symmetrically permute the rows and columns of $A$ in such a way that row/column $i$ is ordered as the $\phi\left(v_{i}\right)$ th row/column in the permuted matrix $P A P^{T}$.

Algorithm 2 shows the basic steps of the recursive moHP algorithm. This algorithm takes a hypergraph $\mathcal{H}=(\mathcal{V}, \mathcal{N})$ and fixed-vertex sets $\mathcal{F}_{L} \subseteq \mathcal{V}$ and $\mathcal{F}_{R} \subseteq \mathcal{V}$ as input and returns an $m^{\prime}$-way ordered partition of $\mathcal{H}$, where $m^{\prime}$ denotes the number of free vertices in $\mathcal{H}$. Note that $m^{\prime}=m$ for the initial invocation of this algorithm. The base case and the recursive step of the moHP algorithm are covered in lines 1-2 and 3-8, respectively. In the base case, i.e., when there is exactly one free vertex in $\mathcal{V}$, the singleton partition $\left\langle v_{i}\right\rangle$ is returned, where $v_{i}$ denotes that free vertex. In the recursive step, i.e., when there are multiple free vertices in $\mathcal{V}$, an ordered bipartition $\Pi=\left\langle\mathcal{V}_{L}, \mathcal{V}_{R}\right\rangle$ of $\mathcal{H}$ is first obtained. In this bipartitioning, the objective is to minimize the left-cut-net metric (5), which is to be explained in section 4.2. The $\epsilon$ value to be used in this bipartitioning (see (2)) is investigated in section 5 . After $\Pi$ is obtained, the FORM algorithm is invoked in order to form new hypergraphs $\mathcal{H}_{L}=\left(\mathcal{V}_{L}, \mathcal{N}_{L}\right)$ and $\mathcal{H}_{R}=\left(\mathcal{V}_{R}, \mathcal{N}_{R}\right)$ as well as new fixed-vertex sets $\mathcal{F}_{L R}$ and $\mathcal{F}_{R L}$. The details of the FORM algorithm are given in section 4.3. Then, the moHP algorithm is recursively invoked on hypergraphs $\mathcal{H}_{L}$ and $\mathcal{H}_{R}$ to respectively obtain an $m_{L}^{\prime}$-way ordered partition $\Pi_{m o}^{L}$ of $\mathcal{H}_{L}$ and an $m_{R}^{\prime}$-way ordered partition $\Pi_{m o}^{R}$ of $\mathcal{H}_{R}$, where $m_{L}^{\prime}$ and $m_{R}^{\prime}$, respectively, denote the numbers of free vertices in $\mathcal{H}_{L}$ and $\mathcal{H}_{R}$. Here, $m^{\prime}=m_{L}^{\prime}+m_{R}^{\prime}$. Finally, by concatenating $\Pi_{m o}^{L}$ and $\Pi_{m o}^{R}$, an $m^{\prime}$-way ordered partition $\Pi_{m o}$ of $\mathcal{H}$ is obtained and returned.

As seen in the recursive invocations of the moHP algorithm in lines 6 and 7 , the old fixed-vertex sets $\mathcal{F}_{L}$ and $\mathcal{F}_{R}$ associated with the current hypergraph $\mathcal{H}$ are inherited by the new hypergraphs $\mathcal{H}_{L}$ and $\mathcal{H}_{R}$. That is, the left-fixed-vertex set $\mathcal{F}_{L}$ and the right-fixed-vertex set $\mathcal{F}_{R}$ of $\mathcal{H}$ become the left-fixed-vertex set of $\mathcal{H}_{L}$ and the right-fixed-vertex set of $\mathcal{H}_{R}$, respectively. In other words, the vertices that become 


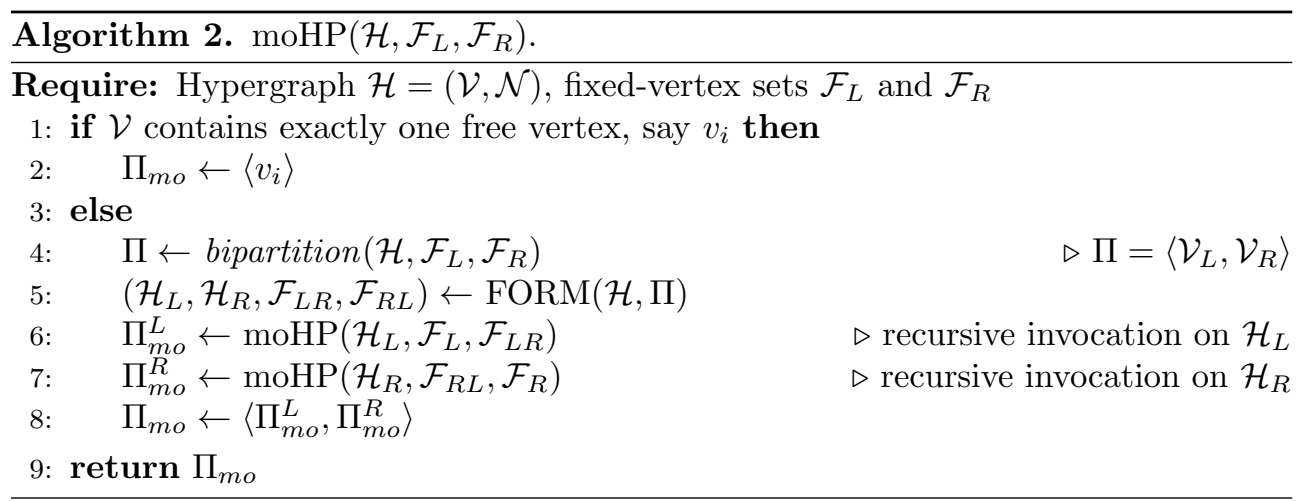

(net $n_{b}$ : cut, but not left-cut)

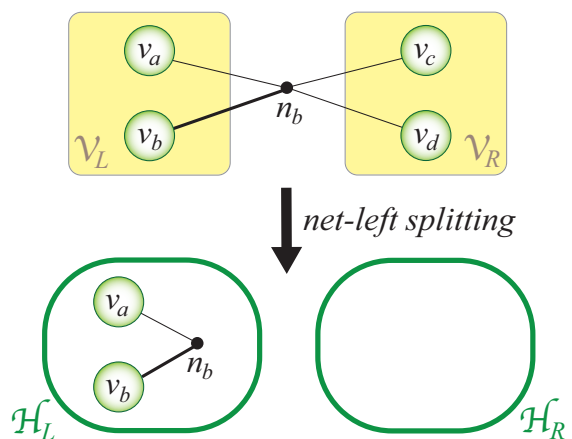

(net $n_{g}$ : left-cut)

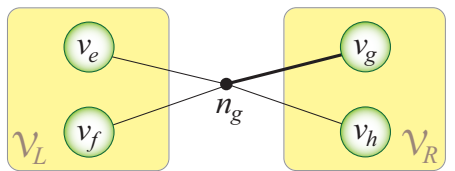

net duplication

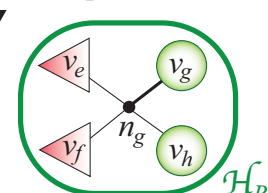

FIG. 3. Upper part: Cut nets $n_{b}$ and $n_{g}$. Net $n_{b}$ is not left-cut since $v_{b} \in \mathcal{V}_{L}$, whereas net $n_{g}$ is left-cut since $v_{g} \in \mathcal{V}_{R}$. Lower part: Net-left splitting and net duplication are applied on $n_{b}$ and $n_{g}$, respectively.

fixed to the left/right part in an invocation of the moHP algorithm remain fixed to the left/right part in further recursive invocations.

4.2. Left-cut-net metric. Consider the ordered bipartition $\Pi=\left\langle\mathcal{V}_{L}, \mathcal{V}_{R}\right\rangle$ obtained in line 4 of Algorithm 2. Recall that a cut net is defined as a net connecting multiple parts. For encoding the minimization objective of the moHP problem in individual bipartitioning steps, we introduce a special type of cut net, which is referred to as left-cut net. A net $n_{i}$ is said to be a left-cut net if $v_{i}$ is assigned to $\mathcal{V}_{R}$ and at least one pin of $n_{i}$ is assigned to $\mathcal{V}_{L}$. Figure 3 displays sample cut nets, $n_{b}$ and $n_{g}$, where $n_{g}$ is a left-cut net while $n_{b}$ is not.

The set of the left-cut nets, which is denoted by $\mathcal{N}_{\ell c}$, is formulated as

$$
\mathcal{N}_{\ell c}=\left\{n_{i}: \operatorname{Pins}\left(n_{i}\right) \cap \mathcal{V}_{L} \neq \emptyset \text { and } v_{i} \in \operatorname{Pins}\left(n_{i}\right) \cap \mathcal{V}_{R}\right\} .
$$

While obtaining the ordered bipartition $\Pi$ of $\mathcal{H}$, the objective is to minimize the left-cut-net metric, which is defined as the number of left-cut nets in П, i.e.,

$$
\text { left-cut-net }(\Pi)=\left|\mathcal{N}_{\ell c}\right| \text {. }
$$

Section 4.4 shows the correctness of this bipartitioning objective in terms of minimizing the cost of the $m$-way ordered partition obtained by the moHP algorithm, whereas section 4.5 describes how existing partitioning tools can be utilized for encapsulating this bipartitioning objective. 
4.3. Forming $\mathcal{H}_{L}$ and $\mathcal{H}_{R}$ by novel cut-net manipulation techniques. Algorithm 3 displays the basic steps of the FORM algorithm. As input, it takes a hypergraph $\mathcal{H}=(\mathcal{V}, \mathcal{N})$ and an ordered bipartition $\Pi=\left\langle\mathcal{V}_{L}, \mathcal{V}_{R}\right\rangle$ of $\mathcal{H}$, and it returns new hypergraphs $\mathcal{H}_{L}$ and $\mathcal{H}_{R}$ with fixed-vertex sets $\mathcal{F}_{L R}$ and $\mathcal{F}_{R L}$. This algorithm goes over each net $n_{i}$ in $\mathcal{N}$ and depending on the distribution of the pins of $n_{i}$ in $\Pi$, it includes $n_{i}$ in either net set $\mathcal{N}_{L}$ or net set $\mathcal{N}_{R}$ or both. If $n_{i}$ is internal to $\mathcal{V}_{L}$ (i.e., $\operatorname{Pins}\left(n_{i}\right) \subseteq \mathcal{V}_{L}$ ), then it is included in $\mathcal{N}_{L}$ as is. Similarly, if $n_{i}$ is internal to $\mathcal{V}_{R}$ (i.e., $\left.\operatorname{Pins}\left(n_{i}\right) \subseteq \mathcal{V}_{R}\right)$, then it is included in $\mathcal{N}_{R}$ as is. The moHP algorithm handles the cut nets by two novel techniques as follows. If $n_{i}$ is a cut net, but not a left-cut one (i.e., $v_{i} \in \operatorname{Pins}\left(n_{i}\right) \cap \mathcal{V}_{L}$ and Pins $\left(n_{i}\right) \cap \mathcal{V}_{R} \neq \emptyset$ ), then the net-left-splitting technique is applied. In this technique, even though $n_{i}$ has pins in both $\mathcal{V}_{L}$ and $\mathcal{V}_{R}$, it is only included in $\mathcal{N}_{L}$ with its pins that are assigned to $\mathcal{V}_{L}$. If $n_{i}$ is a left-cut net (i.e., $\operatorname{Pins}\left(n_{i}\right) \cap \mathcal{V}_{L} \neq \emptyset$ and $\left.v_{i} \in \operatorname{Pins}\left(n_{i}\right) \cap \mathcal{V}_{R}\right)$, then the net-duplication technique is applied. In this technique, $n_{i}$ is copied to both $\mathcal{N}_{L}$ and $\mathcal{N}_{R}$ with its complete pin set despite the fact that neither $\mathcal{V}_{L}$ nor $\mathcal{V}_{R}$ genuinely contains all of $n_{i}$ 's pins. In lines 12 and 13 of the algorithm, leftpins and rightpins denote the sets of the pins of $n_{i}$ in $\mathcal{V}_{L}$ and $\mathcal{V}_{R}$, respectively. The vertices in rightpins are added to vertex set $\mathcal{V}_{L}$ and they are fixed to the right part of $\mathcal{H}_{L}$, i.e., included in $\mathcal{F}_{L R}$. In a dual manner, the vertices in leftpins are added to vertex set $\mathcal{V}_{R}$ and they are fixed to the left part of $\mathcal{H}_{R}$, i.e., included in $\mathcal{F}_{R L}$. After all nets in $\mathcal{N}$ are considered, new hypergraphs $\mathcal{H}_{L}$ and $\mathcal{H}_{R}$ are formed by $\mathcal{H}_{L}=\left(\mathcal{V}_{L}, \mathcal{N}_{L}\right)$ and $\mathcal{H}_{R}=\left(\mathcal{V}_{R}, \mathcal{N}_{R}\right)$, respectively. As in $\mathcal{H}$, each net in $\mathcal{H}_{L}$ and $\mathcal{H}_{R}$ is assigned a unit cost. Each free vertex in $\mathcal{H}_{L}$ and $\mathcal{H}_{R}$ is assigned a unit weight, whereas each fixed vertex is assigned a zero weight. Finally, hypergraphs $\mathcal{H}_{L}$ and $\mathcal{H}_{R}$ and fixed-vertex sets $\mathcal{F}_{L R}$ and $\mathcal{F}_{R L}$ are returned.

Figure 3 illustrates an example for each of the net-left-splitting and net-duplication techniques. In the figures throughout the paper, fixed vertices are denoted by triangles pointing a direction, whereas free vertices are denoted by circles. Each vertex fixed to the left part is denoted by a triangle pointing left, whereas each vertex fixed to the right part is denoted by a triangle pointing right. Note that for any net $n_{i}$, vertex $v_{i}$ is special compared to the other pins of $n_{i}$ since the part assignment of $v_{i}$ determines whether cut net $n_{i}$ is left-cut or not. Therefore, the connection of $n_{i}$ to $v_{i}$ is drawn thicker in the figures for any net $n_{i}$.

Figure 4 displays an example for the moHP algorithm run on the hypergraph given in Figure 2. In Figure 4, each rectangular shape with a green border and a white background denotes a hypergraph to be bipartitioned during the moHP algorithm, whereas each rectangular shape with a yellow background denotes a part in an ordered bipartition (color available online). To be able to refer to the individual hypergraphs, we label them with a MATLAB like notation according to their coverage on the parts of the resulting $m$-way ordered partition. For example, the initial hypergraph $\mathcal{H}$ is labeled with $\mathcal{H}_{1: 8}$ since it covers all eight parts in the resulting $m$-way ordered partition, while the left and right hypergraphs obtained by bipartitioning $\mathcal{H}_{1: 8}$ are labeled with $\mathcal{H}_{1: 4}$ and $\mathcal{H}_{5: 8}$, respectively. Each left-cut net in the figure is shown in a gray background. Consider the ordered bipartition $\Pi$ of $\mathcal{H}_{1: 8}$. Note that nets $n_{1}, n_{3}$, and $n_{4}$ are cut in $\Pi$, whereas only $n_{3}$ is left-cut among them. Then, left-cut-net $(\Pi)=$ 1 for this bipartition. Since $n_{1}$ and $n_{4}$ are cut but not left-cut, the net-left-splitting technique is applied on them, that is, they are only included in the left hypergraph $\mathcal{H}_{L}=\mathcal{H}_{1: 4}$ with their respective pins assigned to the left part $\mathcal{V}_{L}$. Since $n_{3}$ is leftcut, the net duplication technique is applied on it, that is, $n_{3}$ is included in both hypergraphs $\mathcal{H}_{L}=\mathcal{H}_{1: 4}$ and $\mathcal{H}_{R}=\mathcal{H}_{5: 8}$. Due to the net duplication, the vertices in rightpins $=\left\{v_{3}, v_{6}\right\}$ are added to the left hypergraph $\mathcal{H}_{1: 4}$ as right-fixed, whereas the 


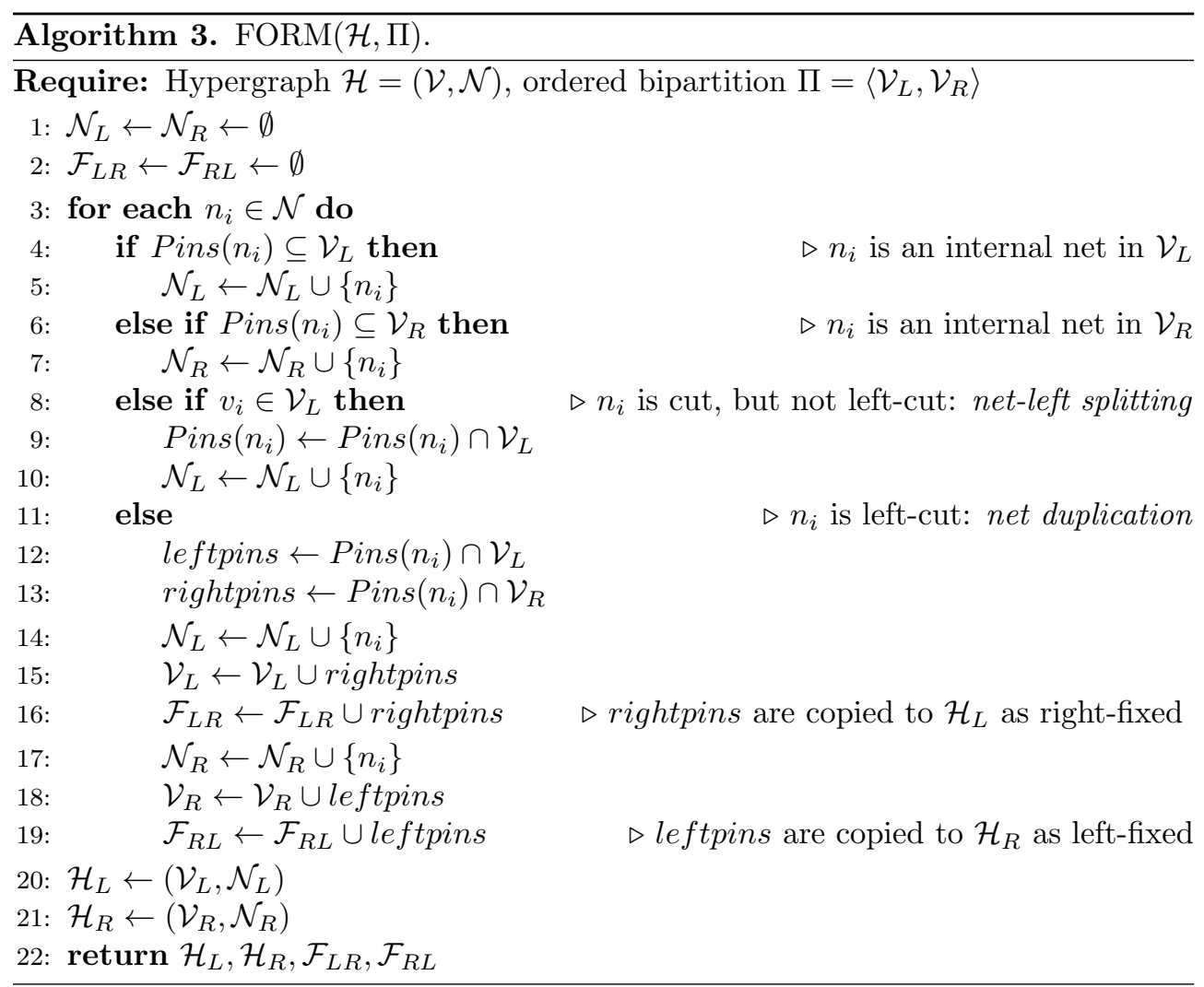

vertices in leftpins $=\left\{v_{4}, v_{1}\right\}$ are added to the right hypergraph $\mathcal{H}_{5: 8}$ as left-fixed.

4.4. Correctness of the moHP algorithm. In this section, Theorem 7 shows that minimizing the left-cut-net metric (5) in each bipartition of the moHP algorithm corresponds to minimizing the cost (4) of resulting $m$-way ordered partition. Before that, we provide a brief discussion on the special pins and give some definitions and lemmas to be used in Theorem 7 .

We first show that $v_{i} \in \operatorname{Pins}\left(n_{i}\right)$ for each net $n_{i}$ during the entire moHP algorithm. Note that $v_{i} \in \operatorname{Pins}\left(n_{i}\right)$ for each net in the initial row-net hypergraph. Consider a net $n_{i}$ in a hypergraph $\mathcal{H}=(\mathcal{V}, \mathcal{N})$ on which the moHP algorithm is invoked and assume that $v_{i} \in \operatorname{Pins}\left(n_{i}\right)$ for each $n_{i} \in \mathcal{N}$. If $n_{i}$ is included in $\mathcal{H}_{L}$ or $\mathcal{H}_{R}$ as is (lines 5 and 7 in Algorithm 3), then $v_{i} \in \operatorname{Pins}\left(n_{i}\right)$ trivially. If net-left splitting is applied on $n_{i}$ (lines 9-10 in Algorithm 3), then $v_{i} \in \operatorname{Pins}\left(n_{i}\right)$ since $v_{i} \in \mathcal{V}_{L}$. If net duplication is applied on $n_{i}$ (lines 12-19 in Algorithm 3), then $v_{i} \in \operatorname{Pins}\left(n_{i}\right)$ for both copies of $n_{i}$ in $\mathcal{H}_{L}$ and $\mathcal{H}_{R}$ since the whole pin set of $n_{i}$ is duplicated to $\mathcal{H}_{L}$ and $\mathcal{H}_{R}$.

For the nets in the moHP algorithm, we introduce four different states that indicate the connections of the nets to fixed vertices. We call a net $n_{i}$

(i) free if it connects no fixed vertices,

(ii) left-anchored if it connects some left-fixed vertices but no right-fixed ones,

(iii) right-anchored if it connects some right-fixed vertices but no left-fixed ones,

(iv) left-right-anchored if it connects some left-fixed and some right-fixed vertices.

Recall that new fixed vertices are only introduced by the net-duplication operation and fixed vertices remain fixed in the descendant invocations of the moHP algorithm. 


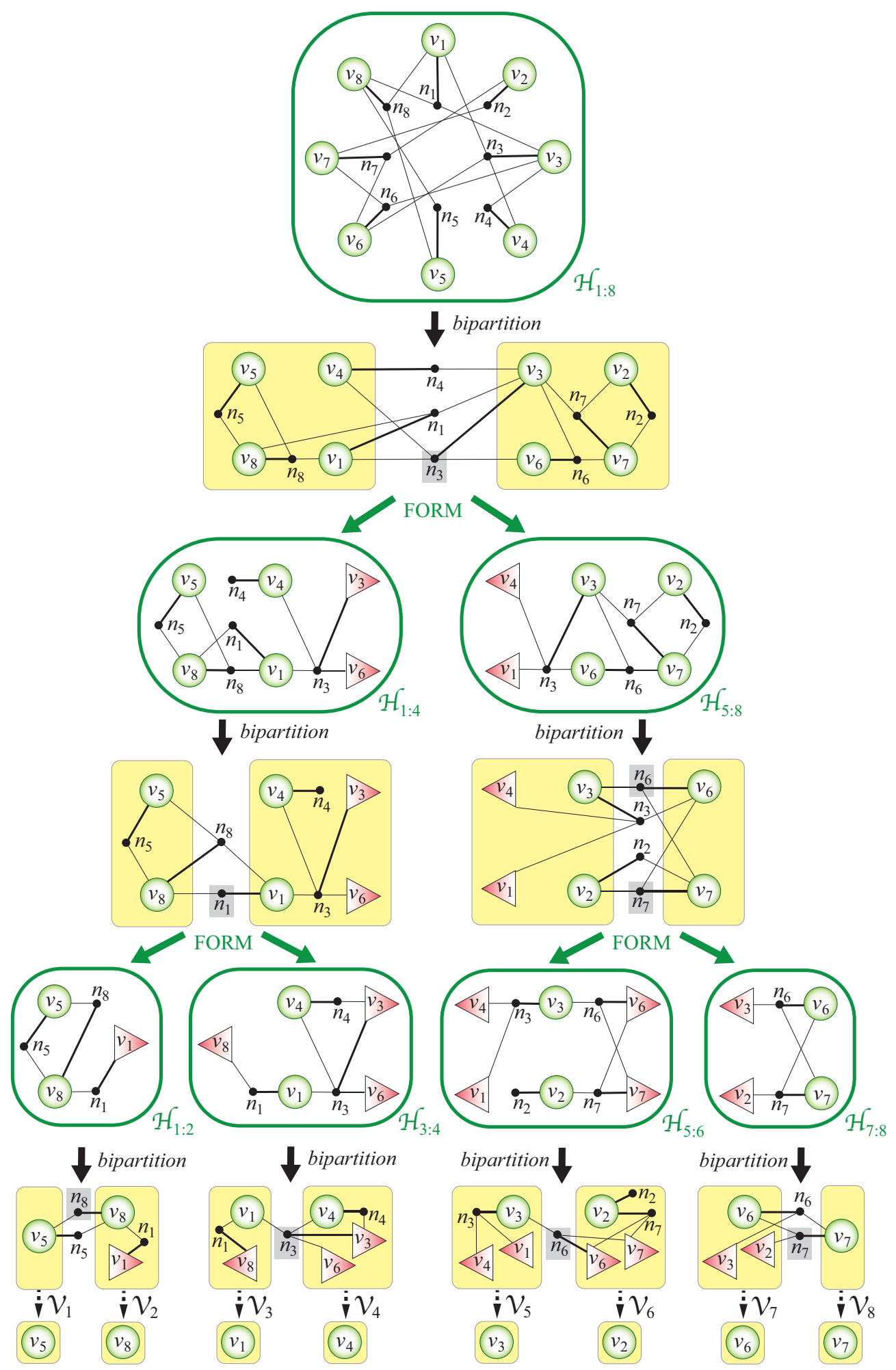

FIG. 4. An example run of the moHP algorithm on the hypergraph given in Figure 2. Left-cut nets are shown in gray background.

Copyright (c) by SIAM. Unauthorized reproduction of this article is prohibited. 


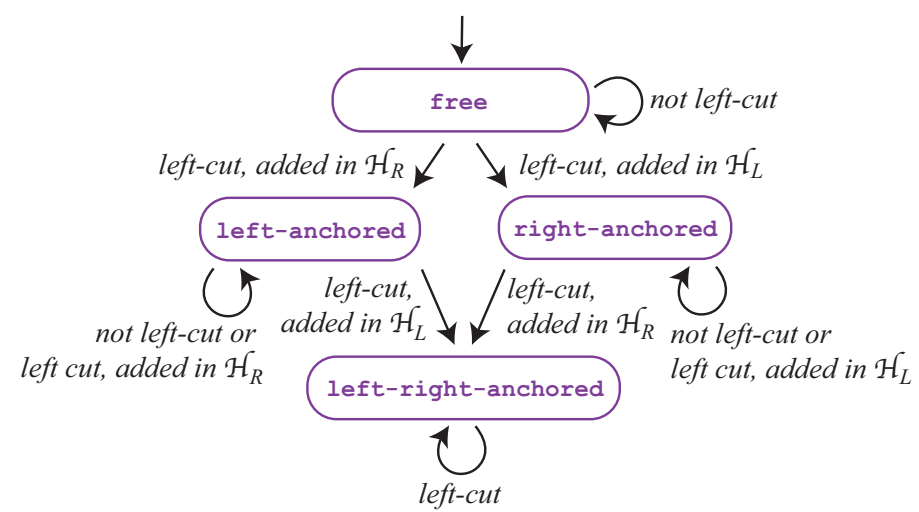

FIG. 5. The state diagram for the states of net $n_{i}$ in the moHP algorithm.

Hence, if a net $n_{i}$ is right-anchored or left-right-anchored, it implies that $n_{i}$ became left-cut in a bipartition performed in an earlier invocation, and among the two copies of $n_{i}$ formed after that bipartition, this copy is the one added to the left hypergraph connecting right-fixed vertices that include $v_{i}$. Therefore, for each right-anchored or left-right-anchored net $n_{i}$, the special pin of $n_{i}$, i.e., $v_{i}$, is among its right-fixed pins. With a dual reasoning, for each free or left-anchored net $n_{i}$, the special pin of $n_{i}$ is among its free pins. Finally, for each free or right-anchored net $n_{i}$, pin $f_{i}$ is among its free pins.

Figure 5 displays a state diagram for the states of a net $n_{i}$ changing through the recursive invocations of the moHP algorithm. Note that all nets are free in the initial invocation of the moHP algorithm; so is $n_{i}$. Since the pins of $n_{i}$ become fixed vertices only after applying net duplication on $n_{i}, n_{i}$ stays free as long as it does not become left-cut. If $n_{i}$ becomes left-cut, net duplication copies it to $\mathcal{H}_{L}$ and $\mathcal{H}_{R}$ so that it becomes right-anchored and left-anchored in $\mathcal{H}_{L}$ and $\mathcal{H}_{R}$, respectively. Similar to the free nets, left-anchored and right-anchored nets do not change their states until they become left-cut. If a left-anchored net $n_{i}$ becomes left-cut, then it becomes left-right-anchored in $\mathcal{H}_{L}$ while remaining left-anchored in $\mathcal{H}_{R}$ after net duplication. In a dual manner, if a right-anchored net $n_{i}$ becomes left-cut, then it becomes leftright-anchored in $\mathcal{H}_{R}$ while remaining right-anchored in $\mathcal{H}_{L}$ after net duplication. Left-right-anchored nets are doomed to become left-cut in all further bipartitionings; hence, a left-right-anchored net $n_{i}$ remains in the same state in both $\mathcal{H}_{L}$ and $\mathcal{H}_{R}$.

The recursive invocations of the moHP algorithm form a hypothetical full binary tree, which is referred to as an $R B$ tree $[1,2,38]$. Each node in the RB tree represents a hypergraph $\mathcal{H}$ on which the moHP algorithm is invoked. If $\mathcal{H}$ contains a single free vertex, which is the base case of the moHP algorithm, then the corresponding node is a leaf node; otherwise, it has one left and one right child node, respectively representing $\mathcal{H}_{L}$ and $\mathcal{H}_{R}$ obtained in line 5 of Algorithm 2. The RB tree rooted at the node corresponding to hypergraph $\mathcal{H}$ is denoted by $\mathcal{T}^{\mathcal{H}}$. Figure 4 displays a sample RB tree with $m=8$ leaf nodes.

Given a net $n_{i}$ in a hypergraph $\mathcal{H}=(\mathcal{V}, \mathcal{N})$ and an RB tree $\mathcal{T}^{\mathcal{H}}$, let $\mu\left(n_{i}, \mathcal{T}^{\mathcal{H}}\right)$ denote the number of bipartitions in $\mathcal{T}^{\mathcal{H}}$ in which $n_{i}$ is left-cut. In the following lemmas and theorem, we abuse the notation and use $\Pi \in \mathcal{T}^{\mathcal{H}}$ to refer to the fact that bipartition $\Pi$ is performed in one of the nodes of $\mathcal{T}^{\mathcal{H}}$. The following lemmas provide the formulation of $\mu\left(n_{i}, \mathcal{T}^{\mathcal{H}}\right)$ for each different state of $n_{i}$. Each of Lemmas 3, 4, 
and 5 is used in the proof(s) of the subsequent lemma(s), whereas Lemma 6 is used in the proof of the theorem. Although we skip the proofs of these lemmas and refer the reader to Appendix A for them, we present all of the lemmas in this section for the sake of completeness. In these lemmas, $\hat{\mathcal{V}}$ denotes the set of free nodes in $\mathcal{H}$, i.e., $\hat{\mathcal{V}}=\mathcal{V}-\left(\mathcal{F}_{L} \cup F_{R}\right)$.

Lemma 3. If $n_{i}$ is left-right-anchored in $\mathcal{H}$, then $\mu\left(n_{i}, \mathcal{T}^{\mathcal{H}}\right)$ is equal to the number of free nodes in $\mathcal{H}$ minus one, that is,

$$
\mu\left(n_{i}, \mathcal{T}^{\mathcal{H}}\right)=|\hat{\mathcal{V}}|-1
$$

Lemma 4. If $n_{i}$ is right-anchored in $\mathcal{H}$, then $\mu\left(n_{i}, \mathcal{T}^{\mathcal{H}}\right)$ is equal to the number of free nodes in $\mathcal{H}$ that are ordered after $f_{i}$ in $\Pi_{\text {mo }}$, that is,

$$
\mu\left(n_{i}, \mathcal{T}^{\mathcal{H}}\right)=\left|\left\{v \in \hat{\mathcal{V}}: \phi(v)>\phi\left(f_{i}\right)\right\}\right| .
$$

Lemma 5. If $n_{i}$ is left-anchored in $\mathcal{H}$, then $\mu\left(n_{i}, \mathcal{T}^{\mathcal{H}}\right)$ is equal to the number of free nodes at $\mathcal{H}$ that are ordered before $v_{i}$ in $\Pi_{m o}$, that is,

$$
\mu\left(n_{i}, \mathcal{T}^{\mathcal{H}}\right)=\left|\left\{v \in \hat{\mathcal{V}}: \phi(v)<\phi\left(v_{i}\right)\right\}\right| .
$$

Lemma 6. If $n_{i}$ is free in $\mathcal{H}$, then $\mu\left(n_{i}, \mathcal{T}^{\mathcal{H}}\right)$ is equal to the number of free nodes in $\mathcal{H}$ that are ordered between $f_{i}$ and $v_{i}$ in $\Pi_{m o}$ inclusive minus one, that is,

$$
\mu\left(n_{i}, \mathcal{T}^{\mathcal{H}}\right)=\left|\left\{v \in \hat{\mathcal{V}}: \phi\left(f_{i}\right) \leq \phi(v) \leq \phi\left(v_{i}\right)\right\}\right|-1 .
$$

THEOREM 7. Consider a hypergraph $\mathcal{H}=(\mathcal{V}, \mathcal{N})$ on which the moHP algorithm is initially invoked, where $\mathcal{V}=\left\{v_{1}, v_{2}, \ldots, v_{m}\right\}, \mathcal{N}=\left\{n_{1}, n_{2}, \ldots, n_{m}\right\}$, and $v_{i} \in$ $\operatorname{Pins}\left(n_{i}\right)$ for each net $n_{i} \in \mathcal{N}$. Minimizing the left-cut-net metric in each bipartition performed in the moHP algorithm corresponds to minimizing the cost of the resulting m-way ordered partition $\Pi_{\text {mo }}$ of $\mathcal{H}$.

Proof. Consider an $m$-way ordered partition $\Pi_{m o}$ of $\mathcal{H}$ obtained by the moHP algorithm and the left span of a net $n_{i}$ in $\mathcal{H}$. Note that all nets in $\mathcal{H}$ are free; so is $n_{i}$. Recall that $l s\left(n_{i}\right)$ is defined as $\phi\left(v_{i}\right)-\phi\left(f_{i}\right)$ in $(3)$; then,

$$
l s\left(n_{i}\right)=\phi\left(v_{i}\right)-\phi\left(f_{i}\right)=\left|\left\{v \in \mathcal{V}: \phi\left(f_{i}\right) \leq \phi(v) \leq \phi\left(v_{i}\right)\right\}\right|-1 .
$$

Then, by Lemma 6 ,

$$
l s\left(n_{i}\right)=\mu\left(n_{i}, \mathcal{T}^{\mathcal{H}}\right) .
$$

Recall that in (4), $\operatorname{cost}\left(\Pi_{m o}\right)$ is defined as the sum of the left spans of the nets in $\mathcal{H}$; then by $(6)$,

$$
\operatorname{cost}\left(\Pi_{m o}\right)=\sum_{n_{i} \in \mathcal{N}} l s\left(n_{i}\right)=\sum_{n_{i} \in \mathcal{N}} \mu\left(n_{i}, \mathcal{T}^{\mathcal{H}}\right) .
$$

Since $\mu\left(n_{i}, \mathcal{T}^{\mathcal{H}}\right)$ is equal to the number of bipartitions in $\mathcal{T}^{\mathcal{H}}$ in which $n_{i}$ is left-cut, it can also be expressed as

$$
\mu\left(n_{i}, \mathcal{T}^{\mathcal{H}}\right)=\sum_{\Pi \in \mathcal{T}^{\mathcal{H}}: n_{i} \in \mathcal{N}_{\ell c}^{\Pi}} 1
$$

Copyright $@$ by SIAM. Unauthorized reproduction of this article is prohibited. 


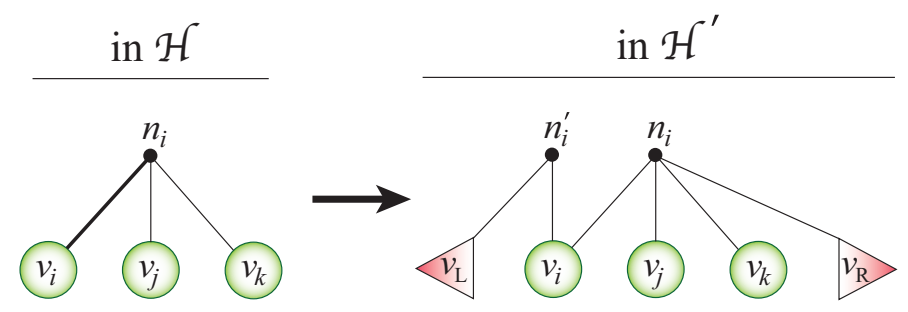

FIG. 6. Net $n_{i}$ in $\mathcal{H}$ and the net pair $\left(n_{i}^{\prime}, n_{i}\right)$ added to $\mathcal{H}^{\prime}$ for $n_{i}$.

Here, $\mathcal{N}_{l c}^{\Pi}$ denotes the set of left-cut nets in $\Pi$. Then, $\operatorname{cost}\left(\Pi_{m o}\right)$ can be formulated as

$$
\begin{aligned}
& \operatorname{cost}\left(\Pi_{m o}\right)=\sum_{n_{i} \in \mathcal{N}} \mu\left(n_{i}, \mathcal{T}^{\mathcal{H}}\right)=\sum_{n_{i} \in \mathcal{N}} \sum_{\Pi \in \mathcal{T}_{\mathcal{H}}: n_{i} \in \mathcal{N}_{\ell c}^{\Pi}} 1=\sum_{\Pi \in \mathcal{T}^{\mathcal{H}}} \sum_{n_{i} \in \mathcal{N}_{\ell c}^{\Pi}} 1 \\
& =\sum_{\Pi \in \mathcal{T}_{\mathcal{H}}} \text { left-cut-net }(\Pi) \text {. }
\end{aligned}
$$

Since $\operatorname{cost}\left(\Pi_{m o}\right)=\sum_{\Pi \in \mathcal{T}_{\mathcal{H}}}$ left-cut-net $(\Pi)$, minimizing the left-cut-net metric in each bipartition in the moHP algorithm corresponds to minimizing the cost of the resulting $m$-way partition.

4.5. Minimizing the left-cut-net metric. Currently, no existing tool is able to bipartition a given hypergraph with the objective of minimizing the left-cut-net metric (5). For this reason, in this section, we formulate the bipartitioning problem with the objective of minimizing the left-cut-net metric as an ordinary hypergraph bipartitioning problem with the objective of minimizing the usual cutsize (1).

Let $\mathcal{H}=(\mathcal{V}, \mathcal{N})$ be a hypergraph which is bipartitioned in line 4 of Algorithm 2 . We first transform $\mathcal{H}$ into an extended hypergraph which is denoted by $\mathcal{H}^{\prime}=\left(\mathcal{V}^{\prime}, \mathcal{N}^{\prime}\right)$. In this transformation, we introduce new vertices $v_{L}$ and $v_{R}$ to the extended vertex set $\mathcal{V}^{\prime}$ in addition to the existing ones in $\mathcal{V}$. That is, $\mathcal{V}^{\prime}=\mathcal{V} \cup\left\{v_{L}, v_{R}\right\}$. Vertices $v_{L}$ and $v_{R}$ are, respectively, fixed to the left and right parts; so the fixed-vertex sets $\mathcal{F}_{L}^{\prime}$ and $\mathcal{F}_{R}^{\prime}$ of $\mathcal{H}^{\prime}$ are obtained from the fixed-vertex sets $\mathcal{F}_{L}$ and $\mathcal{F}_{R}$ of $\mathcal{H}$ by $\mathcal{F}_{L}^{\prime}=\mathcal{F}_{L} \cup\left\{v_{L}\right\}$ and $\mathcal{F}_{R}^{\prime}=\mathcal{F}_{R} \cup\left\{v_{R}\right\}$, respectively. Moreover, for each net $n_{i} \in \mathcal{N}$, we add an updated version of $n_{i}$ and a new net $n_{i}^{\prime}$ to the extended net set $\mathcal{N}^{\prime}$. Net $n_{i}$ is updated by the addition of $v_{R}$ to its pin set, that is, Pins $\left(n_{i}\right) \leftarrow \operatorname{Pins}\left(n_{i}\right) \cup\left\{v_{R}\right\}$. The new net $n_{i}^{\prime}$ connects only $v_{i}$ and $v_{L}$, that is, $\operatorname{Pins}\left(n_{i}^{\prime}\right)=\left\{v_{i}, v_{L}\right\}$. Figure 6 displays an example net $n_{i}$ in $\mathcal{H}$ and the net pair $\left(n_{i}, n_{i}^{\prime}\right)$ added to $\mathcal{H}^{\prime}$ for $n_{i}$.

A bipartition $\Pi^{\prime}=\left\langle\mathcal{V}_{L}^{\prime}, \mathcal{V}_{R}^{\prime}\right\rangle$ of the extended hypergraph $\mathcal{H}^{\prime}$ can be decoded as a bipartition $\Pi=\left\langle\mathcal{V}_{L}, \mathcal{V}_{R}\right\rangle$ of $\mathcal{H}$ by simply removing the newly added vertices $v_{L}$ and $v_{R}$ from $\Pi^{\prime}$. Note that $v_{L} \in \mathcal{V}_{L}^{\prime}$ and $v_{R} \in \mathcal{V}_{R}^{\prime}$ due to being fixed to the respective part; hence, $\mathcal{V}_{L}=\mathcal{V}_{L}^{\prime}-\left\{v_{L}\right\}$ and $\mathcal{V}_{R}=\mathcal{V}_{R}^{\prime}-\left\{v_{R}\right\}$. The following theorem shows the correspondence between the cutsize (1) of the bipartition $\Pi^{\prime}$ of the extended hypergraph $\mathcal{H}^{\prime}$ and the left-cut-net metric (5) of the ordered bipartition $\Pi=\left\langle\mathcal{V}_{L}, \mathcal{V}_{R}\right\rangle$ of $\mathcal{H}$.

THEOREM 8. Let $\mathcal{H}=(\mathcal{V}, \mathcal{N})$ be a hypergraph which is bipartitioned in line 4 of Algorithm 2, and let $\mathcal{H}^{\prime}=\left(\mathcal{V}^{\prime}, \mathcal{N}^{\prime}\right)$ be the corresponding extended hypergraph. Consider a bipartition $\Pi^{\prime}=\left\langle\mathcal{V}_{L}^{\prime}, \mathcal{V}_{R}^{\prime}\right\rangle$ of $\mathcal{H}^{\prime}$ and the bipartition $\Pi=\left\langle\mathcal{V}_{L}, \mathcal{V}_{R}\right\rangle$ of $\mathcal{H}$ induced by $\Pi^{\prime}$. Then, minimizing the cutsize of the bipartition $\Pi^{\prime}(1)$ corresponds to minimizing the left-cut-net metric in $\Pi(5)$. 
Proof. We first show the following:

- both $n_{i}$ and $n_{i}^{\prime}$ are cut in $\Pi^{\prime}$ if $n_{i}$ is left-cut in $\Pi$ (Case 1),

- one of $n_{i}$ and $n_{i}^{\prime}$ is cut in $\Pi^{\prime}$ otherwise (Case 2).

Case 1. Assume that $n_{i}$ is left-cut in $\Pi$. Then, $v_{i} \in V_{R}$ and there exists $v_{j} \in$ $\operatorname{Pins}\left(n_{i}\right)$ such that $v_{j} \in \mathcal{V}_{L}$. It is clear that $j \neq i$. Then, $v_{i} \in V_{R}^{\prime}$ and $v_{j} \in V_{L}^{\prime}$ in $\Pi^{\prime}$. Thus, $n_{i}^{\prime}$ is cut in $\Pi^{\prime}$, since it connects both $\mathcal{V}_{L}^{\prime}$ and $\mathcal{V}_{R}^{\prime}$, respectively, due to pins $v_{L} \in \mathcal{V}_{L}^{\prime}$ and $v_{i} \in \mathcal{V}_{R}^{\prime}$. Similarly, $n_{i}$ is cut in $\Pi^{\prime}$ since it connects both $\mathcal{V}_{L}^{\prime}$ and $\mathcal{V}_{R}^{\prime}$, respectively, due to pins $v_{j} \in \mathcal{V}_{L}^{\prime}$ and $v_{i} \in \mathcal{V}_{R}^{\prime}$.

Case 2.a. Next, assume that $n_{i}$ is not left-cut in $\Pi$ and $v_{i} \in \mathcal{V}_{L}$. Then, $v_{i} \in \mathcal{V}_{L}^{\prime}$ in $\Pi^{\prime}$. Thus, $n_{i}^{\prime}$ is not cut in $\Pi^{\prime}$, since it connects only $\mathcal{V}_{L}^{\prime}$; i.e., both of its pins $\left(v_{i}\right.$ and $v_{L}$ ) reside in $\mathcal{V}_{L}^{\prime}$. On the other hand, $n_{i}$ is cut in $\Pi^{\prime}$, since it connects both $\mathcal{V}_{L}^{\prime}$ and $\mathcal{V}_{R}^{\prime}$, respectively, due to pins $v_{i} \in \mathcal{V}_{L}^{\prime}$ and $v_{R} \in \mathcal{V}_{R}^{\prime}$.

Case 2.b. Finally, assume that $n_{i}$ is not left-cut in $\Pi$ and $v_{i} \in \mathcal{V}_{R}$. If there existed any pins of $n_{i}$ in $\mathcal{V}_{L}$, then $n_{i}$ would be left-cut; hence, all pins of $n_{i}$ reside in $\mathcal{V}_{R}$ in $\Pi$. Note that $v_{R}$ is added to the pin set of $n_{i}$ in $\mathcal{H}^{\prime}$ and $v_{R} \in \mathcal{V}_{R}^{\prime}$. Then $n_{i}$ is not cut in $\Pi^{\prime}$, since all pins of $n_{i}$ reside in $\mathcal{V}_{R}^{\prime}$. On the other hand, $n_{i}^{\prime}$ is cut in $\Pi^{\prime}$, since it connects both $\mathcal{V}_{L}^{\prime}$ and $\mathcal{V}_{R}^{\prime}$, respectively, due to pins $v_{L} \in \mathcal{V}_{L}^{\prime}$ and $v_{i} \in \mathcal{V}_{R}^{\prime}$.

Since there exist two cut nets in $\Pi^{\prime}$ for each left-cut net in $\Pi$, and one cut net in $\Pi^{\prime}$ for each other net in $\mathcal{H}$, the cutsize of $\Pi^{\prime}$ is equal to the left-cut-net metric in $\Pi$ plus the number of nets in $\mathcal{H}$, that is,

$$
\operatorname{cutsize}\left(\Pi^{\prime}\right)=\text { left-cut-net }(\Pi)+|N| \text {. }
$$

Since $|\mathcal{N}|$ is fixed, minimizing the cutsize of $\Pi^{\prime}$ (1) corresponds to minimizing the left-cut-net metric in $\Pi(5)$.

Figure 7 displays hypergraph $\mathcal{H}_{5: 8}$ given in Figure 4, its extended hypergraph $\mathcal{H}_{5: 8}^{\prime}$, a bipartition $\Pi^{\prime}$ of $\mathcal{H}_{5: 8}^{\prime}$, and the bipartition $\Pi$ of $\mathcal{H}_{5: 8}$ induced by $\Pi^{\prime}$. In this figure, the left-cut nets in $\Pi$ and their corresponding cut nets in $\Pi^{\prime}$ are shown in a gray background. Observe that cutsize $\left(\Pi^{\prime}\right)=6$, where left-cut-net $(\Pi)=2$ and $|\mathcal{N}|=4$; hence, cutsize $\left(\Pi^{\prime}\right)=|N|+$ left-cut-net $(\Pi)$.

5. Experiments. In this section, we provide the implementation details of the proposed moHP algorithm and the experimental results that compare the performance of the moHP algorithm against those of the state-of-the-art profile reduction algorithms on an extensive dataset. Our experiments are threefold:

- sensitivity-analysis experiments that compare six different parameter settings for the moHP algorithm in terms of profile and runtime (section 5.3),

- experiments that compare the moHP algorithm against three baseline algorithms in terms of profile and runtime (section 5.4), and

- experiments that compare the moHP algorithm against the best baseline algorithm in terms of the factorization performance in a direct sparse solver (section 5.5).

All experiments are conducted on a Linux workstation equipped with four 18-core CPUs (Intel Xeon Processor E7-8860 v4) and 256 GB of memory.

5.1. Implementation. Recall that in the proposed moHP algorithm, recursion stops when the current hypergraph contains exactly one free vertex. However, in our implementation, we allow the flexibility of early stopping when the number of free vertices in the current hypergraph is smaller than or equal to a threshold, which is denoted by $t$. We refer to this scheme as early stopping. Note that early stopping with $t=1$ is equivalent to the original moHP algorithm. Using $t>1$ results in 


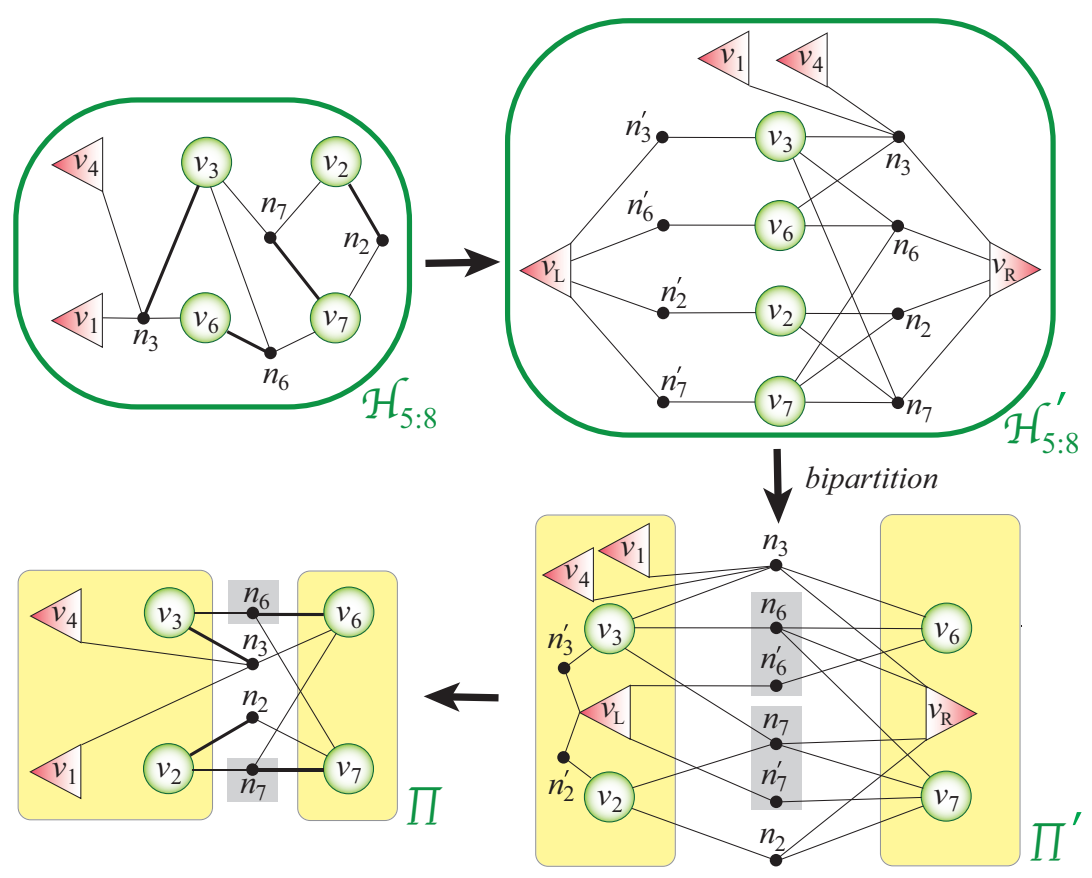

FIG. 7. $\mathcal{H}_{5: 8}$ in Figure 4 , its extended hypergraph $\mathcal{H}_{5: 8}^{\prime}$, bipartition $\Pi^{\prime}$ of $\mathcal{H}_{5: 8}^{\prime}$, and bipartition $\Pi$ of $\mathcal{H}_{5: 8}$ induced by $\Pi^{\prime}$.

an ordered partition with multiple vertices in each part. This partition induces a partial permutation on the rows/columns of the input matrix in such a way that the rows/columns corresponding to the vertices in part $\mathcal{V}_{k}$ are ordered before those corresponding to the vertices in part $\mathcal{V}_{k+1}$ and after those corresponding to the vertices in part $\mathcal{V}_{k-1}$. In order to determine the internal orderings of the resulting row/column blocks, we adapt and use the weighted greed heuristic proposed for profile reduction in [27]. In the original version of this heuristic, a row/column which maximizes a weight function is selected at each iteration and ordered in the right/bottom of the matrix. In our algorithm, we run this heuristic once for each row/column block so that the selection only considers the rows/columns inside the corresponding block.

The motivation for early stopping is that the quality of the bipartitions obtained by multilevel partitioning tools on very small hypergraphs may not always be worth the total runtime of these many bipartitionings on small hypergraphs. Early stopping enables us to exploit the trade-off between the quality and the runtime of the proposed algorithm. Note that the early-stopping scheme with $t=\alpha$ saves at least $\log \alpha$ recursion levels from incurring bipartitioning overhead while losing the merit of performing these unrealized recursion levels. Hence, using a larger threshold results in a faster reordering with a larger profile. The experimental results that compare the performance of the moHP algorithm for varying threshold values are given in section 5.3.

Since the ultimate goal of the proposed model is to obtain an ordering rather than a balanced partitioning, we use a loose balance constraint, i.e., a large $\epsilon$ value in (2), in the bipartitionings performed in the proposed algorithm. Using a looser constraint widens the solution space and hence is likely to result in a better quality. The experimental results that compare the performance of the moHP algorithm for 
varying $\epsilon$ values are given in section 5.3.

The proposed algorithm is implemented in $\mathrm{C}$ and compiled using gcc version 4.9.2 with optimization level two. All source code is available for download. ${ }^{1}$ In each bipartitioning step, $\mathrm{PaToH}$ is used as the hypergraph partitioner. In the preliminary experiments, we observed that the performance of the proposed algorithm varies with the parameters of PaToH (see manual [10]) and using Sweep (the vertex visit order), Absorption Matching (the coarsening algorithm), and Kernihgan-Lin (the refinement algorithm) generally gives a better result. Note that the extended hypergraph $\mathcal{H}^{\prime}$ is obtained from each hypergraph $\mathcal{H}$ to be bipartitioned in line 4 of Algorithm 2. In our efficient implementation, the FORM algorithm obtains the extended hypergraphs $\mathcal{H}_{L}^{\prime}$ and $\mathcal{H}_{R}^{\prime}$ directly from the extended hypergraph $\mathcal{H}^{\prime}$, instead of first forming $\mathcal{H}_{L}$ and $\mathcal{H}_{R}$ and then obtaining $\mathcal{H}_{L}^{\prime}$ and $\mathcal{H}_{R}^{\prime}$.

5.2. Dataset. The experiments are conducted on an extensive dataset of symmetric matrices obtained from the SuiteSparse (formerly known as UFL) Sparse Matrix Collection [13]. This dataset is formed by merging the following sets of matrices:

- 131 matrices that are used in the well-known profile reduction works. Since these works were published some time ago, some of these matrices are small by today's standards. These matrices are all symmetric and include

- the 18 matrices in Kumfert and Pothen's collection, which is used in [3, $7,29,30,35,36]$,

- the 8 matrices in the NASA collection, which is used in [3, 27],

- the $44 A A^{T}$ matrices $^{2}$ with more than 1000 rows in the Netlib Linear Programming Problem collection, which is used in [27],

- the 71 matrices with more than 1000 rows in the Harwell-Boeing collection, which is used in $[3,27,29]$.

- 176 symmetric matrices in the SuiteSparse collection with the number of nonzeros between 1,000,000 and 100,000,000, excluding the ones whose problem type is "graph."

Duplicate matrices are excluded from the dataset; that is, only one of the matrices with the same sparsity pattern is kept in the dataset. An error is encountered when HSL code MA67, which is included in the tested baseline algorithms, is run on eight matrices (boyd1, c-73, boyd2, lp1, c-big, ins2, TSOPF_FS_b39_c30, and mip1); hence those eight matrices are excluded from the dataset as well. The resulting dataset ${ }^{3}$ consists of 295 matrices.

5.3. Sensitivity analysis. In this section, we analyze the effects of the following parameters (mentioned in section 5.1) on the resulting profile and runtime of the moHP algorithm:

- $t$ : threshold value for early stopping, and

- $\epsilon$ : maximum imbalance ratio allowed in each bipartitioning (2). Note that $0 \leq \epsilon \leq 1$ for a bipartition.

We test four different $t$ values $(1,25,250$, and 2500) and two different $\epsilon$ values (0.50 and 0.90); hence the number of compared parameter settings is eight. These experiments are conducted on the dataset of 295 matrices described in section 5.2.

Figure 8 displays two performance profile plots [17] which compare the eight different parameter settings for the moHP algorithm. In these plots, label $\epsilon A-t B$

\footnotetext{
${ }^{1}$ https://github.com/seheracer/profilereduction

${ }^{2} A A^{T}$ is performed using MATLAB.

${ }^{3}$ https://github.com/seheracer/profilereduction/blob/master/dataset
} 


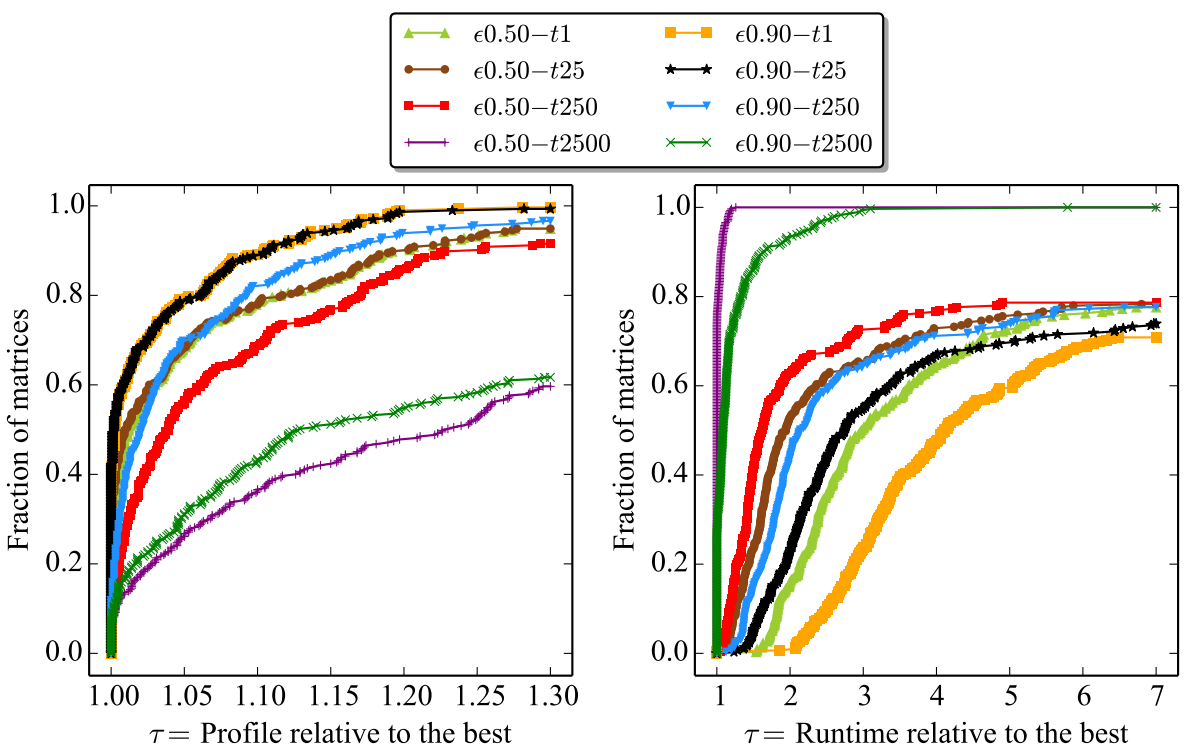

FIG. 8. Performance profile plots comparing the eight versions of the moHP algorithm in terms of profile and runtime.

refers to using $\epsilon=A$ and $t=B$. The plot on the left compares these eight settings in terms of profile, whereas the plot in the right compares them in terms of runtime (of the moHP algorithm). Since we apply the weighted greed heuristic [27] for determining the internal ordering of each row/column block for $t>1$ as mentioned in section 4.5, the runtime values include the runtime of that heuristic as well.

In a performance profile plot [17], the line associated to a method $a$ passing through a point $(\tau, f)$ means that in $100 f \%$ of the instances, the result obtained by $a$ is at most $\tau$ times worse than the best result obtained by the compared methods on the corresponding instance. So, the higher a line is, the better the method associated to that line performs.

In Figure 8, the plot in the left shows that $\epsilon 0.90-t 25$ and $\epsilon 0.90-t 1$ perform the same and outperform the other parameter settings in terms of profile. Observe that for a fixed $\epsilon$ value, using a smaller $t$ value improves profile except for going from $t=25$ to $t=1$. As the $t$ value decreases, the rate of improvement in profile also decreases and converges to zero for $t=1$. This finding is in agreement with the motivation of the early stopping scheme described in section 5.1. Also observe that for a fixed $t$ value, $\epsilon=0.90$ performs better than $\epsilon=0.50$. This can be attributed to the fact that using $\epsilon=0.90$ poses a looser constraint compared to using $\epsilon=0.50$ and hence has a larger solution space, as mentioned in section 5.1. Although we only present the results for $\epsilon=0.50$ and $\epsilon=0.90$, we also tried using $\epsilon=0.70$. Expectedly, the performance of $\epsilon=0.70$ is better than that of $\epsilon=0.50$ but worse than that of $\epsilon=0.90$.

In Figure 8, the plot in the right shows that $\epsilon 0.50-t 2500$ is the fastest setting, whereas $\epsilon 0.90-t 1$ is the slowest one. Observe that using a smaller $t$ value always increases the runtime of the moHP algorithm due to the reasons explained in section 5.1. Using a larger $\epsilon$ value also increases the runtime, which can be attributed 
to the enlarged solution space again.

Considering both of these parameters, one consistent finding is that the runtime of the moHP algorithm increases as the resulting profile decreases. In the experiments given in sections 5.4 and 5.5 , we use $\epsilon 0.90-t 25$ because it is one of the best performers along with $\epsilon 0.90-t 1$ in terms of profile, and it is considerably faster than $\epsilon 0.90-t 1$.

5.4. Comparison against baseline algorithms. In this section, we compare the performance of the moHP algorithm against those of four baseline algorithms, each of which consists of two phases. The heuristics used in these baseline algorithms constitute the state of the art in this field, as also confirmed by $[4,25]$. In the first phase of our baseline algorithms, we use one of the following heuristics: $R C M$ [21], GibbsKing [22], Sloan [40], and HuScott [29]. For RCM, we use the implementation provided by Reid and Scott [35] in HSL code MC60 [12]. For GibbsKing, we use the efficient implementation provided by Lewis [31] in ACM Algorithm 582. For Sloan, we use the enhanced Sloan algorithm provided by Reid and Scott [35] in HSL code MC60 [12]. For HuScott, we use the multilevel hybrid algorithm provided by $\mathrm{Hu}$ and Scott [29] in HSL code MC73 [12]. In the second phase of each baseline algorithm, we use Hager's exchange algorithm [27] provided by Reid and Scott [36] in HSL code MC67 [12], because Reid and Scott [36] report that applying Hager's exchange algorithm as a second phase to certain profile reduction algorithms yields better results than using them separately. Then, the baseline algorithms against which we compare the proposed moHP algorithm are summarized as follows:

- $R C M H$ (RCM+Hager): MC60 with JCNTL(1)=1 followed by MC67.

- GKH (GibbsKing+Hager): The ACM Algorithm 582 followed by MC67.

- $S H$ (Sloan+Hager): MC60 with JCNTL(1)=0 followed by MC67.

- HSH (HuScott+Hager): MC73 followed by MC67.

Each of these codes is used with default setting and compiled with gfortran version 4.9.2 with the -02 optimization flag. The double-precision versions are used for the HSL codes.

Figure 9 displays two performance profile plots comparing the proposed moHP algorithm against the baseline algorithms on the dataset of 295 matrices described in section 5.2. Similar to Figure 8, the one on the left compares them in terms of profile, whereas the one on the right compares them in terms of runtime. As seen in the plot on the left, moHP performs significantly better than each baseline algorithm in terms of profile. This can be attributed to the correct formulation of the profile minimization problem as an moHP problem as well as the solution of this problem via recursive bipartitioning utilizing the successful hypergraph partitioning tool PaToH [10]. Among the baseline algorithms, HSH outperforms the rest and is followed by SH and GKH in order. The plot on the right shows that $\mathrm{SH}$ is the fastest algorithm, followed by HSH and GKH in order. The moHP algorithm, on the other hand, is the slowest algorithm, which can be attributed to the expensive nature of hypergraph partitioning. As will be seen in section 5.5, the quality of the orderings obtained by the moHP algorithm may justify the runtime of the moHP algorithm.

Figure 10 displays eight performance profile plots comparing the proposed moHP algorithm against the baseline algorithms in terms of profile, one for each problem type having at least ten matrices in our dataset of 295 matrices. The title of each plot displays the respective problem type and the number of matrices with that type in parentheses. As seen in the figure, except for types 2D/3D and Structural, the moHP algorithm performs significantly better than the baseline algorithms. In those problem types, moHP is usually followed by HSH, SH, GKH, and RCMH in order. For 


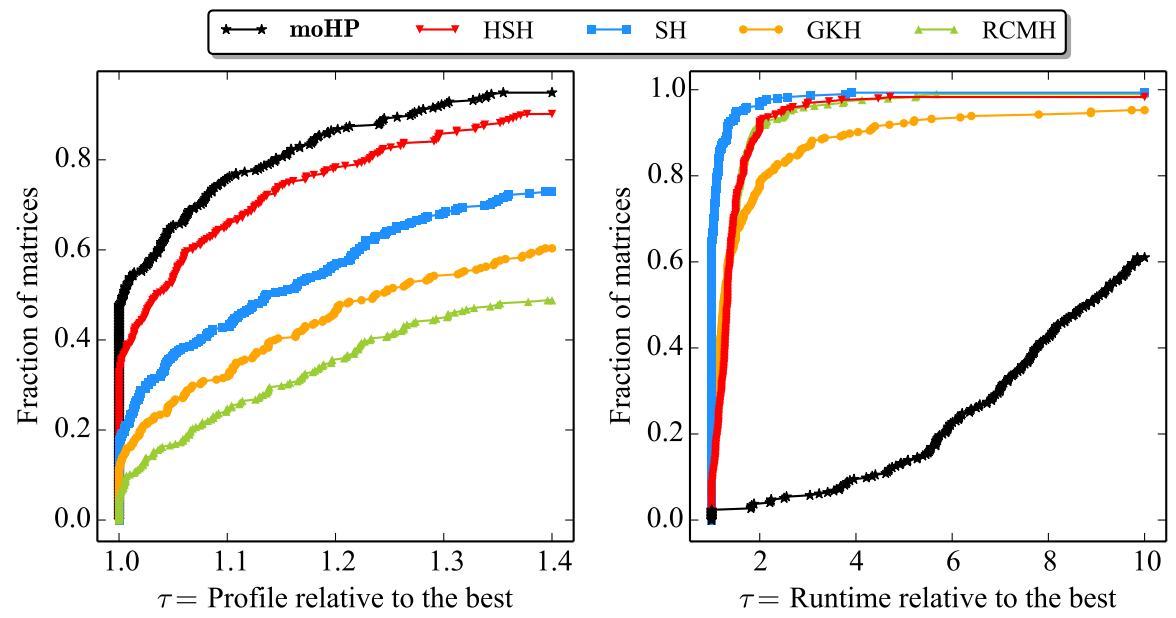

FIG. 9. Performance profile plots comparing the moHP algorithm and the baseline algorithms in terms of profile and runtime.

type Structural, moHP and HSH perform comparably, followed by SH, GKH, and RCMH in order. For type 2D/3D, HSH performs better than all compared algorithms, followed by moHP, $\mathrm{SH}, \mathrm{GKH}$, and $\mathrm{RCMH}$ in order.

5.5. Factorization experiments. In this section, we compare the moHP algorithm only against HSH, which achieves the smallest profile among the baseline algorithms on average. For the evaluation, in addition to the profile and the ordering runtime, we also consider the factorization performance in a sparse solver, HSL code MA57 [12, 18]. MA57 solves sparse symmetric system(s) of linear equations by using a direct multifrontal method, which is based on a sparse variant of Gaussian elimination. We run MA57 on the matrices reordered by HSH and moHP with default settings, and the ordering of each given matrix is kept as is by setting ICNTL(6)=1. The reader is referred to the manual ${ }^{4}$ for the details of MA57. It is compiled with gfortran version 4.9.2 and ATLAS BLAS version 3.11.11.

We consider the following performance metrics obtained during the factorization, i.e., MA57BD:

- Storage: The number of entries in factors (in millions), i.e., INFO(15) $/ 10^{6}$.

- FLOP count: the number of floating-point operations for the elimination (in billions), i.e., RINFO (4) $/ 10^{9}$.

- Runtime: The runtime of MA57BD (in seconds).

We perform the MA57 experiments on a dataset containing only large matrices, derived from the dataset given in section 5.2. First, we included all matrices in the initial dataset with number of rows between 100,000 and 500,000. Then, we excluded each matrix whose factorization (MA57BD) takes longer than six hours when the subject matrix is reordered by HSH. The resulting dataset contains 32 matrices whose numbers of nonzeros range between $1,423,116$ and $32,886,208$. The properties of those matrices and the performance results obtained on them are given in Table 1. In this table, the matrices are sorted in the increasing order of the profile obtained

\footnotetext{
${ }^{4}$ http://www.hsl.rl.ac.uk/specs/ma57.pdf
} 

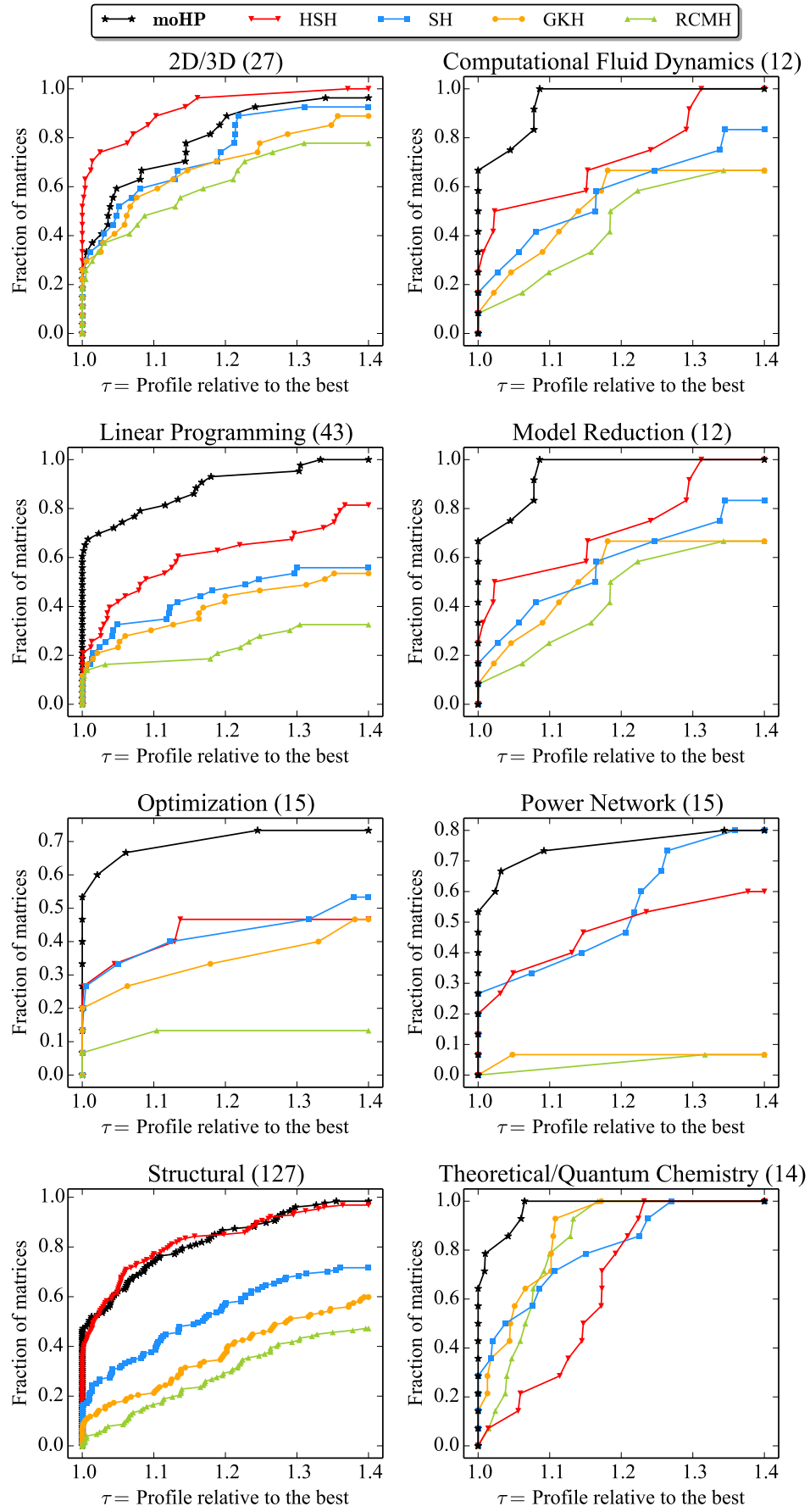

FIG. 10. Performance profile plots comparing the moHP algorithm and the baseline algorithms in terms of profile for different problem types. (.) denotes the number of matrices in the respective problem type.

Copyright (c) by SIAM. Unauthorized reproduction of this article is prohibited. 
by HSH.

Table 1 displays the properties of the 32 test matrices and the results obtained by HSH and moHP on these matrices. Columns 1, 2, and 3, respectively, display the matrix name, the number of rows/columns $(m)$, and the number of nonzeros $(n n z)$. Columns 4-7 display the ordering results, whereas columns 8-13 display the MA57 results. Column pairs $4-5$ and $6-7$, respectively, denote profile and ordering runtime. Column pairs 8-9, 10-11, and 12-13, respectively, denote storage, FLOP count, and runtime of MA57BD. In each column pair, we compare the performances of HSH and moHP in the respective metric and show the better result in boldface on each matrix. Note that column pair 6-7 displays the runtime of the ordering algorithm, whereas column pair 12-13 displays the runtime of the factorization when the respective matrix is reordered by the corresponding algorithm.

As seen in Table 1, HSH performs better than moHP in terms of profile on matrices with small profile, i.e., those on which HSH obtains profile smaller than $230 \times 10^{6}$, except for matrices filter3D, d_pretok, and 2cubes_sphere. In this set of matrices with small profile, although moHP obtains a larger profile than HSH on bmwcra_1 and a comparable profile on shipsec8 and shipsec1, it obtains smaller MA57BD runtime than $\mathrm{HSH}$ on these matrices. On all the matrices with large profile, i.e., those on which HSH obtains a profile larger than $230 \times 10^{6}$, moHP performs better than HSH except for Lin.

As seen in Table 1, HSH runs faster than moHP on all matrices except for Lin. However, when we consider the total runtime, which can be expressed as the sum of the ordering and factorization runtimes, moHP performs better than HSH on the matrices with large profile except for Lin. For example, consider the largest matrix among those given in Table 1, which is dielFilterV3clx with 420,408 rows/columns and $32,886,208$ nonzeros. The ordering runtime of moHP on this largest matrix is 102.3 seconds, which is the highest ordering runtime of the moHP algorithm in the given dataset. Even on this matrix, the total runtimes of HSH and moHP, respectively, are $11.9+953.3=965.2$ seconds and $102.3+743.3=845.6$ seconds, so moHP performs $965.2 / 845.6=1.14 \mathrm{x}$ better than HSH in terms of the total runtime. Similarly, consider the matrix with the largest profile, which is SiO2 with 155,331 rows/columns and $11,283,503$ nonzeros. On this matrix, the total runtimes of $\mathrm{HSH}$ and moHP, respectively, are $11.0+20,719.7=20,730.7$ seconds and $62.7+15,324.9=15,387.6$ seconds, so moHP performs $20,730.7 / 15,387.6=1.35 \mathrm{x}$ better than HSH in terms of the total runtime. Hence, for the matrices with large profile, the better but slower orderings obtained by the moHP algorithm generally pay off very well since they significantly reduce the factorization runtimes.

6. Conclusion. We formulated the profile minimization problem as a constrained version of the hypergraph partitioning (HP) problem, which we refer to as the $m$-way ordered hypergraph partitioning (moHP) problem. For solving the moHP problem, we proposed the moHP algorithm, which utilizes the recursive bipartitioning approach. The moHP algorithm addresses the minimization objective of the moHP problem by utilizing fixed vertices and two novel cut-net manipulation techniques. We theoretically showed the correctness of the proposed moHP algorithm and described how the existing partitioning tools can be utilized in the moHP algorithm. We tested the performance of the moHP algorithm against the state-of-the-art profile reduction algorithms on a large dataset of 295 matrices, and the experimental results showed 


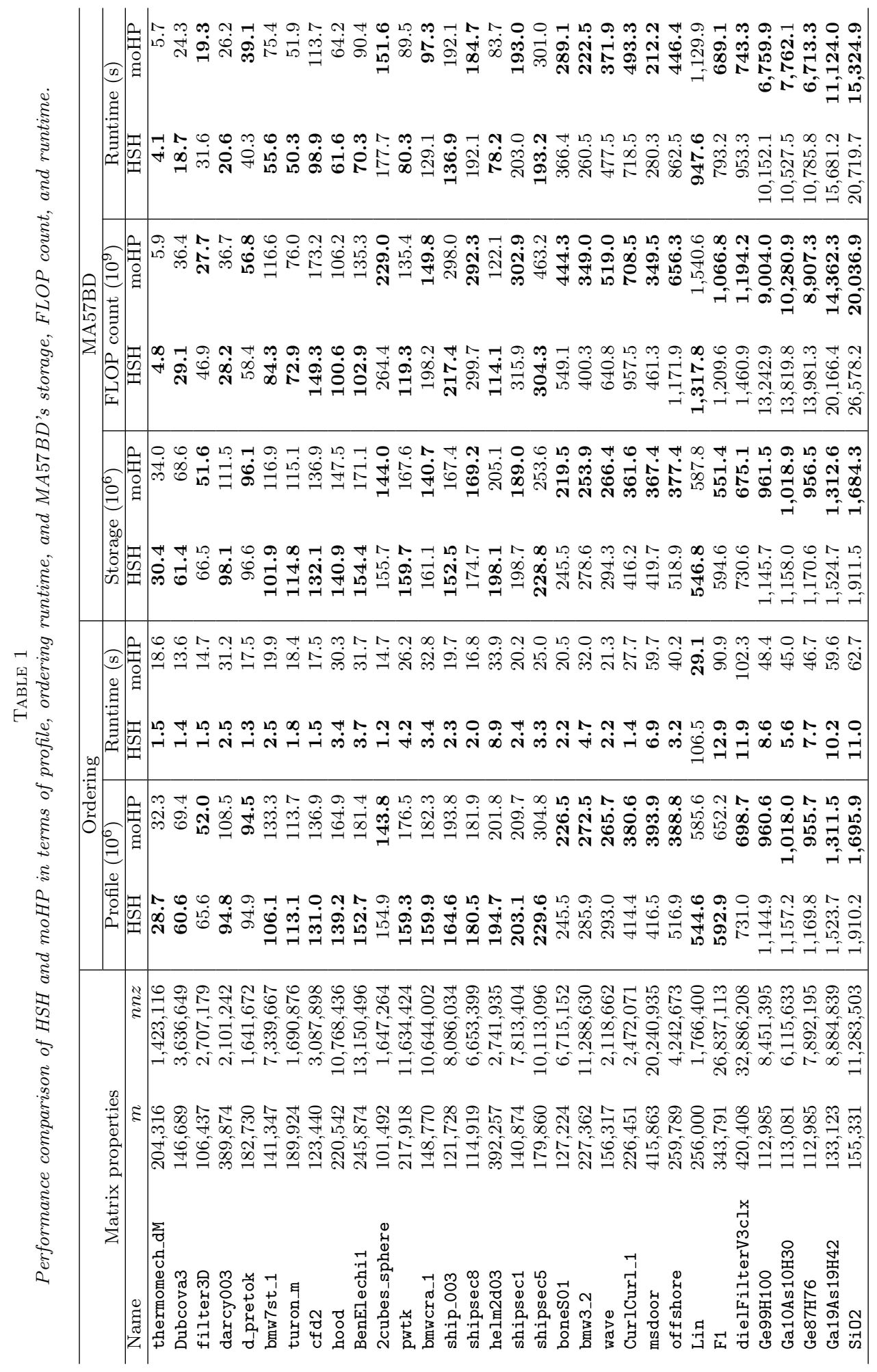

Copyright (c) by SIAM. Unauthorized reproduction of this article is prohibited. 
the validity of the proposed approach.

Appendix A. Proofs of lemmas. We prove each of Lemmas 3, 4, 5, and 6 by induction on the depth of the RB tree. We assume that the depth of $\mathcal{T}^{\mathcal{H}}$ is $k>0$. One important observation is that the depths of subtrees $\mathcal{T}^{\mathcal{H}_{L}}$ and $\mathcal{T}^{\mathcal{H}_{R}}$ are both less than $k$. The base case for the induction in each proof corresponds to the depth of $\mathcal{T}^{\mathcal{H}}$ being equal to zero, which implies that $\mathcal{H}$ is represented by a leaf node. In this case, no further moHP invocations are carried on; hence, $\mu\left(n_{i}, \mathcal{T}^{\mathcal{H}}\right)=0$.

In the following proofs, we use $\hat{\mathcal{V}}, \hat{\mathcal{V}}_{L}$, and $\hat{\mathcal{V}}_{R}$ to denote the number of free vertices in $\mathcal{H}, \mathcal{H}_{L}$, and $\mathcal{H}_{R}$, respectively.

\section{A.1. Lemma 3.}

Proof. In the base case, $\hat{\mathcal{V}}-1=0$ since there is exactly one free vertex in $\mathcal{H}$; hence, $\mu\left(n_{i}, \mathcal{T}^{\mathcal{H}}\right)=|\hat{\mathcal{V}}|-1$ holds.

We assume $\mu\left(n_{i}, \mathcal{T}^{\mathcal{H}}\right)=|\hat{\mathcal{V}}|-1$ holds when the tree depth is less than $k$. Since $n_{i}$ is left-right-anchored, it is left-cut in the bipartition of $\mathcal{H}$. Thus, $n_{i}$ is copied to both $\mathcal{H}_{L}$ and $\mathcal{H}_{R}$ by the net-duplication technique and it is left-right-anchored in both of them. By the inductive hypothesis, $\mu\left(n_{i}, \mathcal{T}^{\mathcal{H}_{L}}\right)=\left|\hat{\mathcal{V}}_{L}\right|-1$ and $\mu\left(n_{i}, \mathcal{T}^{\mathcal{H}_{R}}\right)=\left|\hat{\mathcal{V}}_{R}\right|-1$. Notice that $\hat{\mathcal{V}}_{L}$ and $\hat{\mathcal{V}}_{R}$ are disjoint and $\hat{\mathcal{V}}=\hat{\mathcal{V}}_{L} \cup \hat{\mathcal{V}}_{R}$. Since $n_{i}$ is left-cut in the bipartition of $\mathcal{H}$, we have

$$
\begin{aligned}
\mu\left(n_{i}, \mathcal{T}^{\mathcal{H}}\right) & =\mu\left(n_{i}, \mathcal{T}^{\mathcal{H}_{L}}\right)+\mu\left(n_{i}, \mathcal{T}^{\mathcal{H}_{R}}\right)+1=\left(\left|\hat{\mathcal{V}}_{L}\right|-1\right)+\left(\left|\hat{\mathcal{V}}_{R}\right|-1\right)+1 \\
& =\left|\hat{\mathcal{V}}_{L}\right|+\left|\hat{\mathcal{V}}_{R}\right|-1=|\hat{\mathcal{V}}|-1 .
\end{aligned}
$$

\section{A.2. Lemma 4 .}

Proof. Recall that $n_{i}$ connects $f_{i}$ in $\mathcal{H}$ since it is right-anchored. In the base case, $\hat{\mathcal{V}}=\left\{f_{i}\right\}$ since there is exactly one free vertex in $\mathcal{H}$. Then, $\left|\left\{v \in \hat{\mathcal{V}}: \phi(v)>\phi\left(f_{i}\right)\right\}\right|=$ 0 ; hence, $\mu\left(n_{i}, \mathcal{T}^{\mathcal{H}}\right)=\left|\left\{v \in \hat{\mathcal{V}}: \phi(v)>\phi\left(f_{i}\right)\right\}\right|$ holds.

We assume $\mu\left(n_{i}, \mathcal{T}^{\mathcal{H}}\right)=\left|\left\{v \in \hat{\mathcal{V}}: \phi(v)>\phi\left(f_{i}\right)\right\}\right|$ holds when the tree depth is less than $k$. We investigate the cases of $n_{i}$ being cut or not in the bipartition of $\mathcal{H}$ as follows.

1. $n_{i}$ is cut: Since $n_{i}$ is right-anchored, it is left-cut. Then, $n_{i}$ is copied to both $\mathcal{H}_{L}$ and $\mathcal{H}_{R}$ by the net-duplication technique and it is right-anchored and left-right-anchored in $\mathcal{H}_{L}$ and $\mathcal{H}_{R}$, respectively. By the inductive hypothesis, $\mu\left(n_{i}, \mathcal{T}^{\mathcal{H}_{L}}\right)=\left|\left\{v \in \hat{\mathcal{V}}_{L}: \phi(v)>\phi\left(f_{i}\right)\right\}\right|$. Moreover, by Lemma 3, $\mu\left(n_{i}, \mathcal{T}^{\mathcal{H}_{R}}\right)=\left|\hat{\mathcal{V}}_{R}\right|-1$. Notice that each vertex in $\hat{\mathcal{V}}_{R}$ is numbered after $f_{i}$ since $f_{i} \in \hat{\mathcal{V}}_{L}$. Since $n_{i}$ is left-cut in the bipartition of $\mathcal{H}$, we have

$$
\begin{aligned}
\mu\left(n_{i}, \mathcal{T}^{\mathcal{H}}\right) & =\mu\left(n_{i}, \mathcal{T}^{\mathcal{H}_{L}}\right)+\mu\left(n_{i}, \mathcal{T}^{\mathcal{H}_{R}}\right)+1 \\
& =\left|\left\{v \in \hat{\mathcal{V}}_{L}: \phi(v)>\phi\left(f_{i}\right)\right\}\right|+\left(\left|\hat{\mathcal{V}}_{R}\right|-1\right)+1 \\
& =\left|\left\{v \in \hat{\mathcal{V}}_{L}: \phi(v)>\phi\left(f_{i}\right)\right\}\right|+\left|\hat{\mathcal{V}}_{R}\right|=\left|\left\{v \in \hat{\mathcal{V}}: \phi(v)>\phi\left(f_{i}\right)\right\}\right| .
\end{aligned}
$$

2. $n_{i}$ is not cut: Since $n_{i}$ is right-anchored, it is internal to the right part, which implies that it appears only in $\mathcal{H}_{R}$. Then, $n_{i}$ is right-anchored in $\mathcal{H}_{R}$. Then, by inductive hypothesis,

$$
\mu\left(n_{i}, \mathcal{T}^{\mathcal{H}}\right)=\mu\left(n_{i}, \mathcal{T}^{\mathcal{H} R}\right)=\left|\left\{v \in \hat{\mathcal{V}}_{R}: \phi(v)>\phi\left(f_{i}\right)\right\}\right|=\left|\left\{v \in \hat{\mathcal{V}}: \phi(v)>\phi\left(f_{i}\right)\right\}\right| .
$$




\section{A.3. Lemma 5.}

Proof. Recall that $n_{i}$ connects $f_{i}$ in $\mathcal{H}$ since it is left-anchored. In the base case, $\hat{\mathcal{V}}=\left\{v_{i}\right\}$ since there is exactly one free vertex. Then, $\left|\left\{v \in \hat{\mathcal{V}}: \phi(v)<\phi\left(v_{i}\right)\right\}\right|=0$; hence, $\mu\left(n_{i}, \mathcal{T}^{\mathcal{H}}\right)=\left|\left\{v \in \hat{\mathcal{V}}: \phi(v)<\phi\left(v_{i}\right)\right\}\right|$ holds.

We assume $\mu\left(n_{i}, \mathcal{T}^{\mathcal{H}}\right)=\left|\left\{v \in \hat{\mathcal{V}}: \phi(v)<\phi\left(v_{i}\right)\right\}\right|$ holds when the depth is less than $k$. We investigate the cases of $n_{i}$ being left-cut or not in the bipartition of $\mathcal{H}$ as follows.

1. $n_{i}$ is left-cut. $n_{i}$ is copied to both $\mathcal{H}_{L}$ and $\mathcal{H}_{R}$ by net duplication and it is left-right-anchored and left-anchored at $\mathcal{H}_{L}$ and $\mathcal{H}_{R}$, respectively. By the inductive hypothesis, $\mu\left(n_{i}, \mathcal{T}_{R}^{\mathcal{H}}\right)=\left|\left\{v \in \hat{\mathcal{V}}_{R}: \phi(v)<\phi\left(v_{i}\right)\right\}\right|$. Moreover, by Lemma $3, \mu\left(n_{i}, \mathcal{T}_{L}^{\mathcal{H}}\right)=|\hat{\mathcal{V}}|-1$. Notice that each vertex in $\hat{\mathcal{V}}_{R}$ is numbered before $v_{i}$ since $v_{i} \in \hat{\mathcal{V}}_{R}$. Since $n_{i}$ is left-cut in the bipartition of $\mathcal{H}$, we have

$$
\begin{aligned}
\mu\left(n_{i}, \mathcal{T}^{\mathcal{H}}\right) & =\mu\left(n_{i}, \mathcal{T}^{\mathcal{H}_{L}}\right)+\mu\left(n_{i}, \mathcal{T}^{\mathcal{H}^{R}}\right)+1 \\
& =\left(\left|\hat{\mathcal{V}}_{L}\right|-1\right)+\left|\left\{v \in \hat{\mathcal{V}}_{R}: \phi(v)<\phi\left(v_{i}\right)\right\}\right|+1 \\
& =\left|\hat{\mathcal{V}}_{L}\right|+\left\{v \in \hat{\mathcal{V}}_{R}: \phi(v)<\phi\left(v_{i}\right)\right\}|=|\left\{v \in \hat{\mathcal{V}}_{R}: \phi(v)<\phi\left(v_{i}\right)\right\} \mid .
\end{aligned}
$$

2. $n_{i}$ is not left-cut: Since $n_{i}$ is left-anchored, $n_{i}$ appears only in $\mathcal{H}_{L}$. Then, $n_{i}$ is left-anchored in $\mathcal{H}_{L}$ and a vertex $v \in \hat{\mathcal{V}}$ is in $\hat{\mathcal{V}}_{L}$ whenever $\phi(v)<\phi\left(v_{i}\right)$. Then, by the inductive hypothesis,

$$
\mu\left(n_{i}, \mathcal{T}^{\mathcal{H}}\right)=\mu\left(n_{i}, \mathcal{T}_{L}^{\mathcal{H}}\right)=\left|\left\{v \in \hat{\mathcal{V}}_{L}: \phi(v)<\phi\left(v_{i}\right)\right\}\right|=\left|\left\{v \in \hat{\mathcal{V}}: \phi(v)<\phi\left(v_{i}\right)\right\}\right| .
$$

\section{A.4. Lemma 6.}

Proof. Recall that both $f_{i}$ and $v_{i}$ are free vertices and connected by $n_{i}$ in $\mathcal{H}$ since $n_{i}$ is free. In the base case, $\hat{\mathcal{V}}=\left\{f_{i}=v_{i}\right\}$ since there is exactly one free vertex. Then, $\left|\left\{v \in \hat{\mathcal{V}}: \phi\left(f_{i}\right) \leq \phi(v) \leq \phi\left(v_{i}\right)\right\}\right|-1=0$; hence, $\mu\left(n_{i}, \mathcal{T}^{\mathcal{H}}\right)=\mid\left\{v \in \hat{\mathcal{V}}: \phi\left(f_{i}\right) \leq \phi(v) \leq\right.$ $\left.\phi\left(v_{i}\right)\right\} \mid$.

We assume $\mu\left(n_{i}, \mathcal{T}^{\mathcal{H}}\right)=\left|\left\{v \in \hat{\mathcal{V}}: \phi\left(f_{i}\right) \leq \phi(v) \leq \phi\left(v_{i}\right)\right\}\right|$ holds when the depth is less than $k$. We investigate the cases of $n_{i}$ being left-cut or not in the bipartition of $\mathcal{H}$ as follows.

1. $n_{i}$ is left-cut: $n_{i}$ is copied to both $\mathcal{H}_{L}$ and $\mathcal{H}_{R}$ by net duplication and it is right-anchored and left-anchored in $\mathcal{H}_{L}$ and $\mathcal{H}_{R}$, respectively. By Lemma 4, $\mu\left(n_{i}, \mathcal{T}^{\mathcal{H}_{L}}\right)=\left|\left\{v \in \hat{\mathcal{V}}_{L}: \phi(v)>\phi\left(f_{i}\right)\right\}\right|$. By Lemma $5, \mu\left(n_{i}, \mathcal{T}^{\mathcal{H}_{R}}\right)=\mid\{v \in$ $\left.\hat{\mathcal{V}}_{R}: \phi(v)<\phi\left(v_{i}\right)\right\} \mid$. Notice that each vertex in $\hat{\mathcal{V}}_{L}$ is numbered before $v_{i}$ since $v_{i} \in \hat{\mathcal{V}}_{R}$. Also notice that each vertex in $\hat{\mathcal{V}}_{R}$ is numbered after $f_{i}$ since $f_{i} \in \hat{\mathcal{V}}_{L}$. Since $n_{i}$ is left-cut in the bipartition of $\mathcal{H}$, we have

$$
\begin{aligned}
\mu\left(n_{i}, \mathcal{T}^{\mathcal{H}}\right) & =\mu\left(n_{i}, \mathcal{T}_{L}^{\mathcal{H}}\right)+\mu\left(n_{i}, \mathcal{T}_{R}^{\mathcal{H}}\right)+1 \\
& =\left|\left\{v \in \hat{\mathcal{V}}_{L}: \phi(v)>\phi\left(f_{i}\right)\right\}\right|+\left|\left\{v \in \hat{\mathcal{V}}_{R}: \phi(v)<\phi\left(v_{i}\right)\right\}\right|+1 \\
& =\left(\left|\left\{v \in \hat{\mathcal{V}}_{L}: \phi(v) \geq \phi\left(f_{i}\right)\right\}\right|-1\right)+\left(\left|\left\{v \in \hat{\mathcal{V}}_{R}: \phi(v) \leq \phi\left(v_{i}\right)\right\}\right|-1\right)+1 \\
& =\left|\left\{v \in \hat{\mathcal{V}}: \phi\left(f_{i}\right) \leq \phi(v) \leq \phi\left(v_{i}\right)\right\}\right|-1 .
\end{aligned}
$$

2. $n_{i}$ is not left-cut: Since $n_{i}$ is free in $\mathcal{H}, n_{i}$ appears in only one of $\mathcal{H}_{L}$ and $\mathcal{H}_{R}$ wherein $n_{i}$ remains free. Consider a vertex $v \in \hat{\mathcal{V}}$ satisfying $\phi\left(f_{i}\right) \leq \phi(v) \leq$ $\phi\left(v_{i}\right)$. If $n_{i} \in \mathcal{H}_{L}, v \in \hat{\mathcal{V}}_{L}$; otherwise, $v \in \hat{\mathcal{V}}_{R}$. Without loss of generality, 
assume that $n_{i}$ appears in only $\mathcal{H}_{L}$. Then, by the inductive hypothesis,

$$
\begin{aligned}
\mu\left(n_{i}, \mathcal{T}^{\mathcal{H}}\right) & =\mu\left(n_{i}, \mathcal{T}_{L}^{\mathcal{H}}\right)=\left|\left\{v \in \hat{\mathcal{V}}_{L}: \phi\left(f_{i}\right) \leq \phi(v) \leq \phi\left(v_{i}\right)\right\}\right|-1 \\
& =\left|\left\{v \in \hat{\mathcal{V}}: \phi\left(f_{i}\right) \leq \phi(v) \leq \phi\left(v_{i}\right)\right\}\right|-1 .
\end{aligned}
$$

Acknowledgment. We thank the anonymous referees for their valuable comments, which helped us substantially improve the presentation of this paper.

\section{REFERENCES}

[1] S. Acer, E. Kayahslan, And C. Aykanat, A recursive bipartitioning algorithm for permuting sparse square matrices into block diagonal form with overlap, SIAM J. Sci. Comput., 35 (2013), pp. C99-C121, https://doi.org/10.1137/120861242.

[2] S. Acer, O. Selvitopi, And C. Aykanat, Improving performance of sparse matrix dense matrix multiplication on large-scale parallel systems, Parallel Comput., 59 (2016), pp. 7196, https://doi.org/10.1016/j.parco.2016.10.001.

[3] S. T. Barnard, A. Pothen, and H. D. Simon, A spectral algorithm for envelope reduction of sparse matrices, Numer. Linear Algebra Appl., 2 (1995), pp. 317-334, https://doi.org/10. 1002/nla.1680020402.

[4] J. A. B. Bernardes And S. L. G. De Oliveira, A systematic review of heuristics for profile reduction of symmetric matrices, Procedia Comput. Sci., 51 (2015), pp. 221-230, https: //doi.org/10.1016/j.procs.2015.05.231.

[5] M. W. Berry, B. Hendrickson, And P. Raghavan, Sparse matrix reordering schemes for browsing hypertext, in Lectures in Applied Mathematics 32, AMS, Providence, RI, 1996, pp. 99-124.

[6] M. E. Bolanos, S. Aviyente, And H. Radha, Graph entropy rate minimization and the compressibility of undirected binary graphs, in 2012 IEEE Statistical Signal Processing Workshop (SSP), IEEE, Washington, DC, 2012, pp. 109-112, https://doi.org/10.1109/ SSP.2012.6319634.

[7] E. G. Boman And B. Hendrickson, A Multilevel Algorithm for Reducing the Envelope of Sparse Matrices, Tech. report SCCM-96-14, Stanford University, Stanford, CA, 1996.

[8] D. Burgess and M. Giles, Renumbering unstructured grids to improve the performance of codes on hierarchical memory machines, Adv. Engrg. Softw., 28 (1997), pp. 189-201, https: //doi.org/10.1016/S0965-9978(96)00039-7.

[9] Ü. V. ÇATALYÜREK AND C. AYKANAT, Hypergraph-partitioning-based decomposition for parallel sparse-matrix vector multiplication, IEEE Trans. Parallel Distributed Syst., 10 (1999), pp. 673-693, https://doi.org/10.1109/71.780863.

[10] Ü. V. Çatalyürek and C. Aykanat, PaToH: A Multilevel Hypergraph Partitioning Tool, Version 3.0, Dept. of Computer Engineering, Bilkent University, Ankara, Turkey, 1999; available at https://www.cc.gatech.edu/ umit/software.html.

[11] S. S. CLIFT AND W.-P. TANG, Weighted graph based ordering techniques for preconditioned conjugate gradient methods, BIT, 35 (1995), pp. 30-47, https://doi.org/10.1007/BF01732977.

[12] Computational Mathematics Group, HSL. A Collection of Fortran Codes for Large Scale Scientific Computation, STFC Rutherford Appleton Laboratory, Chilton, Oxfordshire, England, http://www.hsl.rl.ac.uk/.

[13] T. A. Davis and Y. Hu, The University of Florida Sparse Matrix Collection, ACM Trans. Math. Softw., 38 (2011), 25, http://www.cise.ufl.edu/research/sparse/matrices.

[14] T. A. Davis, S. Rajamanickam, and W. M. Sid-Lakhdar, A survey of direct methods for sparse linear systems, Acta Numer., 25 (2016), pp. 383-566, https://doi.org/10.1017/ S0962492916000076.

[15] E. F. D'Azevedo, P. A. Fonsyth, and W.-P. Tang, Ordering methods for preconditioned conjugate gradient methods applied to unstructured grid problems, SIAM J. Matrix Anal. Appl., 13 (1992), pp. 944-961, https://doi.org/10.1137/0613057.

[16] J. Díaz, J. Petit, And M. Serna, A survey of graph layout problems, ACM Comput. Surv., 34 (2002), pp. 313-356, https://doi.org/10.1145/568522.568523.

[17] E. D. DOlan AND J. J. MoRè, Benchmarking optimization software with performance profiles, Math. Program., 91 (2002), pp. 201-213, https://doi.org/10.1007/s101070100263.

[18] I. S. DuFF, MA57 - a code for the solution of sparse symmetric definite and indefinite systems, ACM Trans. Math. Softw., 30 (2004), pp. 118-144, https://doi.org/10.1145/992200.992202.

Copyright $@$ by SIAM. Unauthorized reproduction of this article is prohibited. 
[19] I. S. Duff and G. A. Meurant, The effect of ordering on preconditioned conjugate gradients, BIT, 29 (1989), pp. 635-657, https://doi.org/10.1007/BF01932738.

[20] C. A. Felippa, Introduction to Finite Element Methods, Department of Aerospace Engineering Sciences and Center for Aerospace Structures, University of Colorado Boulder, 2001.

[21] A. George, Computer Implementation of the Finite Element Method, Ph.D. thesis, Stanford University, Stanford, CA, 1971.

[22] N. E. GibBS, Algorithm 509: A hybrid profile reduction algorithm [F1], ACM Trans. Math. Softw., 2 (1976), pp. 378-387, https://doi.org/10.1145/355705.355713.

[23] N. E. Gibbs, W. G. Poole, Jr., And P. K. Stockmeyer, An algorithm for reducing the bandwidth and profile of a sparse matrix, SIAM J. Numer. Anal., 13 (1976), pp. 236-250, https://doi.org/10.1137/0713023.

[24] S. L. Gonzaga de Oliveira, J. A. B. Bernardes, and G. O. Chagas, An evaluation of reordering algorithms to reduce the computational cost of the incomplete Choleskyconjugate gradient method, Comput. Appl. Math., 37 (2018), pp. 2965-3004, https: //doi.org/10.1007/s40314-017-0490-5.

[25] S. L. Gonzaga de Oliveira, J. A. B. Bernardes, and G. O. Chagas, An evaluation of low-cost heuristics for matrix bandwidth and profile reductions, Comput. Appl. Math., 37 (2018), pp. 1412-1471, https://doi.org/10.1007/s40314-016-0394-9.

[26] P. GRINDROD, Range-dependent random graphs and their application to modeling large smallworld Proteome datasets, Phys. Rev. E, 66 (2002), 066702, https://doi.org/10.1103/ PhysRevE.66.066702.

[27] W. W. HAGER, Minimizing the profile of a symmetric matrix, SIAM J. Sci. Comput., 23 (2002), pp. 1799-1816, https://doi.org/10.1137/S1064827500379215.

[28] D. J. Higham, Unravelling small world networks, J. Comput. Appl. Math., 158 (2003), pp. 6174, https://doi.org/10.1016/S0377-0427(03)00471-0.

[29] Y. F. Hu AND J. A. SCOTT, A multilevel algorithm for wavefront reduction, SIAM J. Sci. Comput., 23 (2001), pp. 1352-1375, https://doi.org/10.1137/S1064827500377733.

[30] G. Kumfert and A. Pothen, Two improved algorithms for envelope and wavefront reduction, BIT, 37 (1997), pp. 559-590, https://doi.org/10.1007/BF02510240.

[31] J. G. Lewis, Implementation of the Gibbs-Poole-Stockmeyer and Gibbs-King algorithms, ACM Trans. Math. Softw., 8 (1982), pp. 180-189, https://doi.org/10.1145/355993.355998.

[32] Y. Lin And J. Yuan, Profile minimization problem for matrices and graphs, Acta Math. Appl. Sin., 10 (1994), pp. 107-112, https://doi.org/10.1007/BF02006264.

[33] J. MeIJer AND J. VAN DE POL, Bandwidth and wavefront reduction for static variable ordering in symbolic reachability analysis, in 2016 NASA Formal Methods Symposium, Springer, Cham, 2016, pp. 255-271, https://doi.org/10.1007/978-3-319-40648-0_20.

[34] C. Mueller, B. Martin, and A. Lumsdaine, A comparison of vertex ordering algorithms for large graph visualization, in 2007 6th International Asia-Pacific Symposium on Visualization (Sydney, NSW, Australia), IEEE, Washington, DC, 2007, pp. 141-148, https: //doi.org/10.1109/APVIS.2007.329289.

[35] J. K. ReID AND J. A. SCOTt, Ordering symmetric sparse matrices for small profile and wavefront, Internat. J. Numer. Methods Engrg., 45 (1999), pp. 1737-1755.

[36] J. K. ReID AND J. A. ScotT, Implementing Hager's exchange methods for matrix profile reduction, ACM Trans. Math. Softw., 28 (2002), pp. 377-391, https://doi.org/10.1145/ 592843.592844.

[37] Y. SAAD, Iterative Methods for Sparse Linear Systems, SIAM, Philadelphia, 2003, https://doi. org $/ 10.1137 / 1.9780898718003$.

[38] O. Selvitopi, S. Acer, And C. Aykanat, A recursive hypergraph bipartitioning framework for reducing bandwidth and latency costs simultaneously, IEEE Trans. Parallel Distributed Syst., 28 (2017), pp. 345-358, https://doi.org/10.1109/TPDS.2016.2577024.

[39] D. Silva, M. Velazco, AND A. Oliveira, Influence of matrix reordering on the performance of iterative methods for solving linear systems arising from interior point methods for linear programming, Math. Methods Oper. Res., 85 (2017), pp. 97-112, https://doi.org/10.1007/ s00186-017-0571-7.

[40] S. W. SLOAN, An algorithm for profile and wavefront reduction of sparse matrices, Internat. J. Numer. Methods Engrg., 23 (1986), pp. 239-251, https://doi.org/10.1002/nme.1620230208.

[41] S. Xu, W. Xue, And H. X. Lin, Performance modeling and optimization of sparse matrixvector multiplication on NVIDIA CUDA platform, J. Supercomput., 63 (2013), pp. 710721, https://doi.org/10.1007/s11227-011-0626-0.

Copyright (c) by SIAM. Unauthorized reproduction of this article is prohibited. 